\begin{tabular}{|c|c|}
\hline \multicolumn{2}{|c|}{ Statistica Sinica Preprint No: SS-2020-0449 } \\
\hline Title & $\begin{array}{l}\text { Weak Signal Identification and Inference in Penalized } \\
\text { Likelihood Models for Categorical Responses }\end{array}$ \\
\hline Manuscript ID & SS-2020-0449 \\
\hline URL & http://www.stat.sinica.edu.tw/statistica/ \\
\hline DOI & $10.5705 /$ ss.202020.0449 \\
\hline Complete List of Authors & $\begin{array}{l}\text { Yuexia Zhang, } \\
\text { Peibei Shi, } \\
\text { Zhongyi Zhu, } \\
\text { Linbo Wang and } \\
\text { Annie Qu }\end{array}$ \\
\hline Corresponding Author & Annie $\mathrm{Qu}$ \\
\hline E-mail & aqu2@uci.edu \\
\hline
\end{tabular}


Statistica Sinica

\title{
WEAK SIGNAL IDENTIFICATION AND INFERENCE IN PENALIZED LIKELIHOOD MODELS FOR CATEGORICAL RESPONSES
}

\author{
${ }^{1}$ Yuexia Zhang, ${ }^{2}$ Peibei Shi, ${ }^{3}$ Zhongyi Zhu, ${ }^{4}$ Linbo Wang and ${ }^{5}$ Annie Qu \\ ${ }^{1,4}$ University of Toronto, ${ }^{2}$ Facebook, ${ }^{3}$ Fudan University, ${ }^{5}$ University of California, Irvine
}

\begin{abstract}
:
Penalized likelihood models are widely used to simultaneously select variables and estimate model parameters. However, the existence of weak signals can lead to inaccurate variable selection, biased parameter estimation, and invalid inference. Thus, identifying weak signals accurately and making valid inferences are crucial in penalized likelihood models. In this paper, we develop a unified approach to identify weak signals and make inferences in penalized likelihood models, including the special case when the responses are categorical. To identify weak signals, we utilize the estimated selection probability of each covariate as a measure of signal strength and formulate a signal identification criterion. To construct confidence intervals, we adopt a two-step inference procedure. Extensive simulation studies show that the proposed two-step inference procedure outperforms several existing methods. We illustrate the proposed method with an application to the Practice Fusion diabetes dataset.
\end{abstract}

Key words and phrases: adaptive Lasso, de-biased method, model selection, post-selection inference

\section{Introduction}

In the big data era, massive data are collected with large-dimensional covariates. However, only a part of covariates might be important. To select the important variables and estimate their effects on the response variable, various penalized likelihood models have been 
proposed, such as the penalized least squares regression model (Tibshirani, 1996; Zou and Hastie, 2005; Tibshirani et al., 2005; Yuan and Lin, 2006; Zou, 2006; Zhang, 2010), penalized logistic regression model (Park and Hastie, 2008; Zhu and Hastie, 2004; Wu et al., 2009), and penalized Poisson regression model (Lambert and Eilers, 2005; Jia et al., 2019).

To achieve model selection consistency or variable screening property for a high-dimensional problem, one common condition is the "beta-min" condition, which requires that the nonzero regression coefficients are sufficiently large (Zhao and Yu, 2006, Huang and Xie, 2007; Van de Geer et al., 2011; Tibshirani, 2011; Zhang and Jia, 2017). Therefore, classical methods for variable selection often focus on strong signals that satisfy such condition. However, if the "beta-min" condition is violated, the important variables and unimportant variables could be inseparable and the true important variables might not be selected even if the sample size goes to infinity (Zhang, 2013). In finite samples, the estimators shrink the true regression coefficients due to the penalty function. When the signal strength is not strong, its coefficient is more likely to be shrunk to zero (Shi and Qu, 2017; Liu et al., 2020). The inaccurate variable selection and biased parameter estimation could lead to a poor post-selection inference, for example, the estimation of confidence intervals could be inaccurate. Thus, weak signals need to be taken into consideration in addition to strong signals. Identification and inference for weak signals can also help discover some potential important variables in practice. For example, in genome-wide association studies (GWAS), some overlooked risk factors for diseases may be recovered by incorporating weak signals (Liu et al., 2020).

For linear regression models, studies have been done on weak signals. In more extreme cases, Jin et al. (2014) assumed all signals were individually weak and proposed graphlet screening for variable selection. Zhang (2017) proposed the perturbed Lasso, where signals 
were strengthened by adding random perturbations to the design matrix. However, these methods only focused on variable selection consistency and did not aim to identify weak signals or provide statistical inference. For weak signal identification and inference, Shi and $\mathrm{Qu}(2017)$ proposed a weak signal identification procedure in finite samples and introduced a two-step inference method to construct confidence intervals after signal identification. However, their derivation relied on a crucial assumption that the design matrix was orthogonal, which may not hold in practice. On the other hand, Li et al. (2019) took advantage of the correlations among covariates and detected weak signals through the partial correlations between strong and weak signals. However, they did not study weak signal inference. Recently, Liu et al. (2020) proposed a method combining the bootstrap Lasso and partial ridge regression for the construction of confidence intervals when there were weak signals in the covariates. However, as stated in their paper, the confidence intervals of the coefficients, with magnitudes of order $1 / \sqrt{n}$, may be invalid.

Moreover, to the best of our knowledge, there has been little work on weak signals in likelihood-based models for categorical responses. One exception is Reangsephet et al. (2020), who proposed variable selection methods for logistic regression models with weak signals. But they did not conduct weak signal identification or inference.

In this paper, we address these gaps by developing a new unified approach to weak signal identification and inference in penalized likelihood models, including the special case when the responses are categorical. Specifically, the estimated probability of each covariate being selected by the one-step adaptive Lasso estimator is used to measure the signal strength. After signal identification, a two-step inference procedure is proposed to construct the confidence intervals for the regression coefficients. The proposed method has several 
advantages. First, we extend the method of Shi and Qu (2017) from linear regression models to likelihood-based models, including generalized linear models. However, our extension is not trivial. For example, in Shi and Qu (2017), the selection probability has an explicit expression. For the proposed likelihood-based method, such an explicit expression does not exist for categorical responses. Thus we propose a new method to estimate the selection probability. Second, in Shi and Qu (2017), the selection probability for the covariate $\boldsymbol{X}_{j}$ is an increasing function of $\left|\beta_{j 0}\right|$, where $\beta_{j 0}$ is the corresponding coefficient of $\boldsymbol{X}_{j}$. Under our current general framework, such a conclusion is not necessarily true. Thus the signal identification criterion in our paper is directly based on the estimated selection probability, which is very different from Shi and Qu (2017). We also discuss how each signal's selection probability is influenced by other covariates due to non-linear modeling or collinearity among covariates; while in Shi and $\mathrm{Qu}(2017)$, the selection probability of one covariate is independent of those of other covariates. Third, Shi and Qu (2017) assumed the design matrix in a linear regression model to be orthogonal, while the proposed method relaxes this constraint. Fourth, the proposed inference method is also different from the inference method in Shi and $\mathrm{Qu}$ (2017). Specifically, we construct the confidence interval for noise variables as well, while their method does not. Simulation results show that our new two-step inference method performs better than the two-step inference method based on the idea of Shi and Qu (2017). In particular, the proposed confidence intervals can achieve accurate coverage probabilities for all signal strength levels.

The remainder of this paper is organized as follows. In Section 2, we introduce the one-step adaptive Lasso estimator and derive the variable selection condition. In Section 3. we propose the weak signal identification criterion. In Section 4 , we develop a two-step 
inference procedure for the construction of confidence intervals. In Section 5, we conduct simulation studies to assess the finite sample performance of the proposed method. In Section 6, we apply the proposed method to the analysis of diabetes data. In Section 7, we provide a brief discussion and concluding remarks. We provide the technical details of proofs, implementation details of several methods, and some additional results in Supplementary Materials.

\section{One-step adaptive Lasso estimator and variable selection condition}

In this section, we introduce the one-step penalized likelihood estimator and derive the condition for variable selection, which will be used later for weak signal identification and inference.

Let $\left(\mathbf{x}_{1}^{\top}, y_{1}\right)^{\top}, \ldots,\left(\mathbf{x}_{n}^{\top}, y_{n}\right)^{\top}$ be $n$ independent and identically distributed random vectors, where $\mathbf{x}_{i}=\left(x_{i 1}, \ldots, x_{i p}\right)^{\top}$ is a $p \times 1$ vector of predictors and $y_{i}$ is a response variable. Assume that $y_{i}$ depends on $\mathbf{x}_{i}$ through a linear combination $\mathbf{x}_{i}^{\top} \boldsymbol{\beta}_{0}$ and the conditional log-likelihood of $y_{i}$ given $\mathbf{x}_{i}$ is $\ell_{i}\left(\boldsymbol{\gamma}_{0}\right)=\ell_{i}\left(\alpha_{0}+\mathbf{x}_{i}^{\top} \boldsymbol{\beta}_{0}, y_{i}\right)$, where $\boldsymbol{\gamma}_{0}=\left(\alpha_{0}, \boldsymbol{\beta}_{0}^{\top}\right)^{\top}, \alpha_{0}$ is an unknown true location parameter and $\boldsymbol{\beta}_{0}=\left(\beta_{10}, \cdots, \beta_{p 0}\right)^{\top}$ is an unknown $p \times 1$ vector of covariate effects. Note that for a likelihood-based model, it is not always possible to eliminate the location parameter via centering the covariates and response variable. For simplicity, assume $p<n$ and $p$ is fixed. Let $\ell(\gamma)=\sum_{i=1}^{n} \ell_{i}(\gamma)$ denote the log-likelihood. Assume $\boldsymbol{\gamma}^{(0)}$ is the maximum likelihood estimator of $\boldsymbol{\gamma}_{0}$, then $\boldsymbol{\gamma}^{(0)}=\left(\alpha^{(0)}, \boldsymbol{\beta}^{(0) \top}\right)^{\top}=\operatorname{argmax}_{\boldsymbol{\gamma}} \ell(\boldsymbol{\gamma})$. In matrix notation, we set $\mathbf{X}=\left(\mathbf{x}_{1}, \ldots, \mathbf{x}_{n}\right)^{\top}=\left(\boldsymbol{X}_{1}, \ldots, \boldsymbol{X}_{p}\right)$ with $\boldsymbol{X}_{j}=\left(x_{1 j}, \ldots, x_{n j}\right)^{\top}$ and $\boldsymbol{Y}=\left(y_{1}, \ldots, y_{n}\right)^{\top}$. Furthermore, denote $\widetilde{\mathbf{x}}_{i}=\left(1, \mathbf{x}_{i}^{\top}\right)^{\top}$ and $\widetilde{\mathbf{X}}=(\mathbf{1}, \mathbf{X})$, where $\mathbf{1}$ is a $n \times 1$ vector with all the elements being 1. Throughout this paper, it is assumed that $\mathrm{E}\left(x_{i j}\right)=0$ and $\operatorname{Var}\left(x_{i j}\right)=1$ for 
all $i \in\{1, \ldots, n\}$ and $j \in\{1, \ldots, p\}$, which can be realized via standardizing the covariate matrix $\mathbf{X}$ in practice.

Assume that some components of $\boldsymbol{\beta}_{0}$ are zero. In order to estimate model parameters and select important variables simultaneously, we consider the penalized likelihood function $\ell(\gamma) / n-\sum_{j=1}^{p} p_{\lambda_{j}}\left(\left|\beta_{j}\right|\right)$, where $p_{\lambda_{j}}(\cdot)$ is a penalty function controlled by the tuning parameter $\lambda_{j}$. One popular penalty function is derived from the adaptive Lasso estimator $(\mathrm{Zou}, 2006)$, where $p_{\lambda_{j}}\left(\left|\beta_{j}\right|\right)=\lambda\left|\beta_{j}\right| /\left|\beta_{j}^{(0)}\right|$. Maximizing the penalized likelihood function is equivalent to minimizing

$$
-\frac{1}{n} \ell(\gamma)+\sum_{j=1}^{p} p_{\lambda_{j}}\left(\left|\beta_{j}\right|\right)
$$

with respect to $\boldsymbol{\gamma}$. According to Wang and Leng (2007) and Zou and Li (2008), if the loglikelihood function has the first and second derivatives, then the log-likelihood function can be approximated through Taylor expansion. Furthermore, the objective function (2.1) can be approximated by

$$
Q_{1}(\boldsymbol{\gamma})=-\frac{1}{2 n}\left(\boldsymbol{\gamma}-\boldsymbol{\gamma}^{(0)}\right)^{\top} \ddot{\ell}\left(\boldsymbol{\gamma}^{(0)}\right)\left(\boldsymbol{\gamma}-\boldsymbol{\gamma}^{(0)}\right)+\sum_{j=1}^{p} p_{\lambda_{j}}\left(\left|\beta_{j}\right|\right)
$$

where $\ddot{\ell}(\cdot)$ is the second derivative of function $\ell(\cdot)$. The one-step penalized likelihood estimator is $\boldsymbol{\gamma}^{(1)}=\left(\alpha^{(1)}, \boldsymbol{\beta}^{(1) \top}\right)^{\top}=\operatorname{argmin}_{\boldsymbol{\gamma}} Q_{1}(\boldsymbol{\gamma})$.

Denote $\mu_{i}(\boldsymbol{\gamma})=\mu_{i}=\widetilde{\mathbf{x}}_{i}^{\top} \boldsymbol{\gamma}$ and $\ell_{i}\left\{\mu_{i}(\boldsymbol{\gamma})\right\}=\ell_{i}\left(\widetilde{\mathbf{x}}_{i}^{\top} \boldsymbol{\gamma}, y_{i}\right)$. Let $\mathbf{D}(\boldsymbol{\gamma})$ be a $n \times n$ diagonal matrix with the $(i, i)$ th element $D_{i i}(\gamma)=-\partial^{2} \ell_{i}\left\{\mu_{i}(\gamma)\right\} / \partial \mu_{i}^{2}, i=1, \ldots, n$. Then $\ddot{\ell}(\gamma)=$ $-\widetilde{\mathbf{X}}^{\top} \mathbf{D}(\boldsymbol{\gamma}) \widetilde{\mathbf{X}}$. Furthermore, we assume $D_{i i}(\boldsymbol{\gamma})$ is a continuous function of $\boldsymbol{\gamma}$. For simplicity, denote $\mathbf{D}\left(\boldsymbol{\gamma}^{(0)}\right), \mathbf{D}\left(\boldsymbol{\gamma}_{0}\right), D_{i i}\left(\boldsymbol{\gamma}^{(0)}\right)$ and $D_{i i}\left(\boldsymbol{\gamma}_{0}\right)$ as $\mathbf{D}^{(0)}, \mathbf{D}_{0}, D_{i i}^{(0)}$ and $D_{0, i i}$, respectively. By 
solving the equation $\partial Q_{1}(\gamma) / \partial \alpha=0$, we can obtain that

$$
\alpha-\alpha^{(0)}=\left(\mathbf{1}^{\top} \mathbf{D}^{(0)} \mathbf{1}\right)^{-1} \mathbf{1}^{\top} \mathbf{D}^{(0)} \mathbf{X}\left(\boldsymbol{\beta}^{(0)}-\boldsymbol{\beta}\right)
$$

Replacing $\alpha-\alpha^{(0)}$ by 2.3$)$ in 2.2 , we obtain the following objective function $Q_{2}(\boldsymbol{\beta})$

$$
\begin{aligned}
Q_{2}(\boldsymbol{\beta}) & =\frac{1}{2 n}\left(\boldsymbol{\beta}-\boldsymbol{\beta}^{(0)}\right)^{\top} \mathbf{X}^{\top} \mathbf{D}^{\dagger(0)} \mathbf{X}\left(\boldsymbol{\beta}-\boldsymbol{\beta}^{(0)}\right)+\sum_{j=1}^{p} p_{\lambda_{j}}\left(\left|\beta_{j}\right|\right) \\
& =\frac{1}{2 n}\left(\boldsymbol{\beta}-\boldsymbol{\beta}^{(0)}\right)^{\top} \mathbf{X}^{\top} \mathbf{D}^{\star(0) \top} \mathbf{D}^{\star(0)} \mathbf{X}\left(\boldsymbol{\beta}-\boldsymbol{\beta}^{(0)}\right)+\sum_{j=1}^{p} p_{\lambda_{j}}\left(\left|\beta_{j}\right|\right),
\end{aligned}
$$

where $\mathbf{D}^{\dagger(0)}=\mathbf{D}^{(0)}-\mathbf{D}^{(0)} \mathbf{1}\left(\mathbf{1}^{\top} \mathbf{D}^{(0)} \mathbf{1}\right)^{-1} \mathbf{1}^{\top} \mathbf{D}^{(0)}$ and $\mathbf{D}^{\star(0)}=\left(\mathbf{D}^{(0)}\right)^{1 / 2}-\left(\mathbf{D}^{(0)}\right)^{1 / 2} \mathbf{1}\left(\mathbf{1}^{\top} \mathbf{D}^{(0)} \mathbf{1}\right)^{-1}$ $\times \mathbf{1}^{\top} \mathbf{D}^{(0)}$. Denote $\mathbf{D}_{0}^{\dagger}=\mathbf{D}_{0}-\mathbf{D}_{0} \mathbf{1}\left(\mathbf{1}^{\top} \mathbf{D}_{0} \mathbf{1}\right)^{-1} \mathbf{1}^{\top} \mathbf{D}_{0}$ and $\mathbf{D}_{0}^{\star}=\mathbf{D}_{0}^{1 / 2}-\mathbf{D}_{0}^{1 / 2} \mathbf{1}\left(\mathbf{1}^{\top} \mathbf{D}_{0} \mathbf{1}\right)^{-1} \mathbf{1}^{\top} \mathbf{D}_{0}$ correspondingly.

In this paper, we mainly focus on weak signal identification using the one-step adaptive Lasso estimator. However, our method can also be extended to other penalized likelihood estimators. Following the idea of Zou and Li (2008), the algorithm for computing the onestep adaptive Lasso estimator $\gamma^{(1)}$ is as follows.

Step 1. Create the working data by $\mathbf{X}^{\star}=\mathbf{D}^{\star(0)} \mathbf{X} \mathbf{W}$ and $\boldsymbol{Y}^{\star}=\mathbf{D}^{\star(0)} \mathbf{X} \boldsymbol{\beta}^{(0)}$, where $\mathbf{W}=$ $\operatorname{diag}\left\{\left|\beta_{1}^{(0)}\right|, \ldots,\left|\beta_{p}^{(0)}\right|\right\}$

Step 2. Apply the coordinate descent algorithm to solve

$$
\hat{\boldsymbol{\beta}}^{\star}=\underset{\boldsymbol{\beta}}{\operatorname{argmin}}\left\{\frac{1}{2 n} \sum_{i=1}^{n}\left(y_{i}^{\star}-\sum_{j=1}^{p} x_{i j}^{\star} \beta_{j}\right)^{2}+\lambda \sum_{j=1}^{p}\left|\beta_{j}\right|\right\},
$$

where $\hat{\boldsymbol{\beta}}^{\star}=\left(\hat{\beta}_{1}^{\star}, \ldots, \hat{\beta}_{p}^{\star}\right)^{\top}, y_{i}^{\star}$ is the $i$ th element of $\boldsymbol{Y}^{\star}$ and $x_{i j}^{\star}$ is the $(i, j)$ th element 


\section{of $\mathbf{X}^{\star}$.}

Step 3. Obtain the value of $\boldsymbol{\beta}^{(1)}=\left(\beta_{1}^{(1)}, \ldots, \beta_{p}^{(1)}\right)^{\top}$ by $\beta_{j}^{(1)}=\hat{\beta}_{j}^{\star}\left|\beta_{j}^{(0)}\right|, \quad j=1 \ldots, p$.

Step 4. Obtain the value of $\alpha^{(1)}$ by $\alpha^{(1)}=\left(\mathbf{1}^{\top} \mathbf{D}^{(0)} \mathbf{1}\right)^{-1} \mathbf{1}^{\top} \mathbf{D}^{(0)} \mathbf{X}\left(\boldsymbol{\beta}^{(0)}-\boldsymbol{\beta}^{(1)}\right)+\alpha^{(0)}$.

From the above algorithm, it can be found that if $\hat{\beta}_{j}^{\star} \neq 0$, then the covariate $\boldsymbol{X}_{j}$ will be selected. According to (2.5), by using the coordinate descent algorithm, we can obtain that

$$
\hat{\beta}_{j}^{\star}=s\left\{\frac{\sum_{i=1}^{n}\left(y_{i}^{\star}-\sum_{k \neq j} x_{i k}^{\star} \hat{\beta}_{k}^{\star}\right) x_{i j}^{\star}}{\sum_{i=1}^{n}\left(x_{i j}^{\star}\right)^{2}}, \frac{n \lambda}{\sum_{i=1}^{n}\left(x_{i j}^{\star}\right)^{2}}\right\},
$$

where $s(z, r)=\operatorname{sgn}(z)(|z|-r)_{+}$. Then the condition for $\hat{\beta}_{j}^{\star} \neq 0\left(\beta_{j}^{(1)} \neq 0\right)$ is

$$
\left|\frac{\sum_{i=1}^{n}\left(y_{i}^{\star}-\sum_{k \neq j} x_{i k}^{\star} \hat{\beta}_{k}^{\star}\right) x_{i j}^{\star}}{\sum_{i=1}^{n}\left(x_{i j}^{\star}\right)^{2}}\right|>\frac{n \lambda}{\sum_{i=1}^{n}\left(x_{i j}^{\star}\right)^{2}} .
$$

For each $i \in\{1, \ldots, n\}$ and $s \in\{1, \ldots, n\}$, let $d_{i s}^{(0)}$ be the $(i, s)$ th element of $\mathbf{D}^{\star(0)}$, then the variable selection condition 2.6 is equivalent to

$$
\begin{array}{r}
\left|\sum_{i=1}^{n}\left(\sum_{s=1}^{n} d_{i s}^{(0)} x_{s j}\right)^{2}\left(\beta_{j}^{(0)}\right)^{2}+\sum_{k \neq j} \sum_{i=1}^{n}\left(\sum_{s=1}^{n} d_{i s}^{(0)} x_{s k}\right)\left(\sum_{s=1}^{n} d_{i s}^{(0)} x_{s j}\right) \beta_{j}^{(0)}\left(\beta_{k}^{(0)}-\beta_{k}^{(1)}\right)\right| \\
>n \lambda .
\end{array}
$$

Similar to the proof in Zou and Li (2008), we can obtain that if the tuning parameter $\lambda$ satisfies the conditions of $\sqrt{n} \lambda \rightarrow 0$ and $n \lambda \rightarrow \infty$, then the one-step adaptive Lasso estimator enjoys the model selection consistency and the non-zero one-step adaptive Lasso estimators 
have the property of asymptotic normality.

\section{Weak signal definition and identification}

\subsection{Weak signal definition}

Suppose a model contains both strong and weak signals. Without loss of generality, assume the covariate matrix $\mathbf{X}$ consists of three components, that is, $\mathbf{X}=\left\{\mathbf{X}^{(S)}, \mathbf{X}^{(W)}, \mathbf{X}^{(N)}\right\}$, where $\mathbf{X}^{(S)}, \mathbf{X}^{(W)}$ and $\mathbf{X}^{(N)}$ represent the subsets of strong signals, weak signals and noise variables, respectively. Following Shi and Qu (2017), we use the selection probability of each covariate to measure the signal strength. Specifically, for any penalized model selection estimator $\hat{\boldsymbol{\beta}}=\left(\hat{\beta}_{1}, \ldots, \hat{\beta}_{p}\right)^{\top}$, we define $P_{d, j}$ as the probability of selecting the covariate $\boldsymbol{X}_{j}$, that is, $P_{d, j}=P\left(\hat{\beta}_{j} \neq 0\right), j \in\{1, \ldots, p\}$. For the one-step adaptive Lasso estimator $\boldsymbol{\beta}^{(1)}=$ $\left(\beta_{1}^{(1)}, \ldots, \beta_{p}^{(1)}\right)^{\top}$, based on the variable selection condition 2.7$), P_{d, j}$ does not have an explicit form. However, in Supplementary Material S1, we show that $P_{d, j}$ can be approximated by $P_{d, j}^{*}$, where

$$
\begin{array}{r}
P_{d, j}^{*}=\Phi\left(\frac{-\sqrt{\frac{\lambda \mathrm{E}\left(D_{0, i i}\right)}{\mathrm{E}\left(D_{0, i i} x_{i j}^{2}\right) \mathrm{E}\left(D_{0, i i}\right)-\left\{\mathrm{E}\left(D_{0, i i} x_{i j}\right)\right\}^{2}}}+\beta_{j 0}}{\sqrt{\left\{\mathrm{E}\left(\widetilde{\mathbf{X}}^{\top} \mathbf{D}_{0} \widetilde{\mathbf{X}}\right)\right\}_{j+1, j+1}^{-1}}}\right) \\
+\Phi\left(\frac{-\sqrt{\frac{\lambda \mathrm{E}\left(D_{0, i i}\right)}{\mathrm{E}\left(D_{0, i i} x_{i j}^{2}\right) \mathrm{E}\left(D_{0, i i}\right)-\left\{\mathrm{E}\left(D_{0, i i} x_{i j}\right)\right\}^{2}}}-\beta_{j 0}}{\sqrt{\left\{\mathrm{E}\left(\widetilde{\mathbf{X}}^{\top} \mathbf{D}_{0} \widetilde{\mathbf{X}}\right)\right\}_{j+1, j+1}^{-1}}}\right) .
\end{array}
$$

Intuitively, in the derivation of selection probability, we can omit the terms of (S2) and (S3) in Supplementary Material S1 and simplify the calculation using asymptotic theory, then we can relax the orthogonality assumption required in Shi and $\mathrm{Qu}(2017)$. We require the following mild assumption to ensure 3.1 is valid. 
Assumption 1. For each $i \in\{1, \ldots, n\}$ and $j \in\{1, \ldots, p\}, P\left(D_{0, i i}>0\right)=1, \mathrm{E}\left(D_{0, i i}\right)<\infty$, $\mathrm{E}\left(D_{0, i i} x_{i j}^{2}\right)<\infty$, and $\mathrm{E}\left(\widetilde{\mathbf{X}}^{\top} \mathbf{D}_{0} \widetilde{\mathbf{X}}\right)$ is positive definite.

The condition of $P\left(D_{0, i i}>0\right)=1$ implies that the conditional log-likelihood function of $y_{i}$ given $\mathbf{x}_{i}, \ell_{i}\left\{\mu_{i}(\gamma)\right\}$, is a concave function of $\mu_{i}(\gamma)$. This is a necessary condition for uniqueness of the maximum likelihood estimator $\gamma^{(0)}$. In addition, according to the CauchySchwarz inequality, this also ensures that $\mathrm{E}\left(D_{0, i i} x_{i j}^{2}\right) \mathrm{E}\left(D_{0, i i}\right)-\left\{\mathrm{E}\left(D_{0, i i} x_{i j}\right)\right\}^{2}>0$. The conditions of $\mathrm{E}\left(D_{0, i i}\right)<\infty$ and $\mathrm{E}\left(D_{0, i i} x_{i j}^{2}\right)<\infty$ guarantee that all expectations of random variables in (3.1) are bounded for finite $n$. The positive definite condition of $\mathrm{E}\left(\widetilde{\mathbf{X}}^{\top} \mathbf{D}_{0} \widetilde{\mathbf{X}}\right)$ is a necessary condition for asymptotic normality of the maximum likelihood estimator $\gamma^{(0)}$, and can ensure $\left\{\mathrm{E}\left(\widetilde{\mathbf{X}}^{\top} \mathbf{D}_{0} \widetilde{\mathbf{X}}\right)\right\}_{j+1, j+1}^{-1}>0$.

To have a deep understanding of $P_{d, j}^{*}$, we first study the asymptotic properties of $P_{d, j}^{*}$. When $\beta_{j 0}=0$,

$$
P_{d, j}^{*}=2 \Phi\left(\frac{-\sqrt{\frac{n \lambda}{\left[\mathrm{E}\left(D_{0, i i} x_{i j}^{2}\right) \mathrm{E}\left(D_{0, i i}\right)-\left\{\mathrm{E}\left(D_{0, i i} x_{i j}\right)\right\}^{2}\right] / \mathrm{E}\left(D_{0, i i}\right)}}}{\sqrt{\left\{\mathrm{E}\left(\widetilde{\mathbf{X}}^{\top} \mathbf{D}_{0} \widetilde{\mathbf{X}}\right) / n\right\}_{j+1, j+1}^{-1}}}\right) .
$$

Under Assumption 1 . $\left[\mathrm{E}\left(D_{0, i i} x_{i j}^{2}\right) \mathrm{E}\left(D_{0, i i}\right)-\left\{\mathrm{E}\left(D_{0, i i} x_{i j}\right)\right\}^{2}\right] / \mathrm{E}\left(D_{0, i i}\right)$ and $\left\{\mathrm{E}\left(\widetilde{\mathbf{X}}^{\top} \mathbf{D}_{0} \widetilde{\mathbf{X}}\right) / n\right\}_{j+1, j+1}^{-1}$ are both positive and bounded. If $n \lambda \rightarrow \infty$, then $P_{d, j}^{*} \rightarrow 0$.

When $\beta_{j 0} \neq 0$,

$$
\begin{array}{r}
P_{d, j}^{*}=\Phi\left(\frac{-\sqrt{n}\left[\sqrt{\frac{\lambda \mathrm{E}\left(D_{0, i i}\right)}{\mathrm{E}\left(D_{0, i i} x_{i j}^{2}\right) \mathrm{E}\left(D_{0, i i}\right)-\left\{\mathrm{E}\left(D_{0, i i} x_{i j}\right)\right\}^{2}}}-\beta_{j 0}\right]}{\sqrt{\left\{\mathrm{E}\left(\widetilde{\mathbf{X}}^{\top} \mathbf{D}_{0} \widetilde{\mathbf{X}}\right) / n\right\}_{j+1, j+1}^{-1}}}\right) \\
+\Phi\left(\frac{-\sqrt{n}\left[\sqrt{\frac{\lambda \mathrm{E}\left(D_{0, i i}\right)}{\mathrm{E}\left(D_{0, i i} x_{i j}^{2}\right) \mathrm{E}\left(D_{0, i i}\right)-\left\{\mathrm{E}\left(D_{0, i i} x_{i j}\right)\right\}^{2}}}+\beta_{j 0}\right]}{\sqrt{\left\{\mathrm{E}\left(\widetilde{\mathbf{X}}^{\top} \mathbf{D}_{0} \widetilde{\mathbf{X}}\right) / n\right\}_{j+1, j+1}^{-1}}}\right) .
\end{array}
$$


If $\sqrt{n} \lambda \rightarrow 0$, then $P_{d, j}^{*} \rightarrow 1$ under Assumption 1 .

These asymptotic properties of $P_{d, j}^{*}$ are consistent with the conclusion that the one-step adaptive Lasso estimator enjoys the property of model selection consistency if $\lambda$ satisfies the conditions of $\sqrt{n} \lambda \rightarrow 0$ and $n \lambda \rightarrow \infty$.

In the following, we study the finite sample properties of $P_{d, j}^{*}$. To illustrate, we first consider three special cases, where the likelihood-based model is taken as a linear regression model, a logistic regression model, and a Poisson regression model, respectively.

\section{Case One: Linear regression model}

We first illustrate the simplest case under the linear regression model setting. Let $y_{i}=$ $\alpha_{0}+\mathbf{x}_{i}^{\top} \boldsymbol{\beta}_{0}+\varepsilon_{i}$, where $\varepsilon_{i} \stackrel{i . i . d .}{\sim} \mathcal{N}\left(0, \sigma^{2}\right)$, then $D_{0, i i}=1 / \sigma^{2}$. If we assume $\operatorname{corr}\left(x_{i j}, x_{i k}\right)=0$ for any $k, k \neq j$, then

$$
P_{d, j}^{*}=\Phi\left(\frac{\beta_{j 0}-\sqrt{\lambda} \sigma}{\sigma / \sqrt{n}}\right)+\Phi\left(\frac{-\beta_{j 0}-\sqrt{\lambda} \sigma}{\sigma / \sqrt{n}}\right)
$$

Note that if the tuning parameter $\lambda$ is replaced by $\lambda_{S h i}=\lambda \sigma^{2}$, then $P_{d, j}^{*}$ has the same form as that in Shi and Qu (2017), where the covariate matrix is assumed to be orthogonal. In this case, $P_{d, j}^{*}$ does not depend on $\gamma_{0}^{-j}$, where $\gamma_{0}^{-j}$ stands for other components in $\gamma_{0}$ except $\beta_{j 0}$. In addition, given any values in $P_{d, j}^{*}$ except $\beta_{j 0}, P_{d, j}^{*}$ is a symmetric function of $\beta_{j 0}$ and increases with $\left|\beta_{j 0}\right|$. Thus, both $P_{d, j}^{*}$ and $\left|\beta_{j 0}\right|$ can be used to measure the signal strength of $\boldsymbol{X}_{j}$, as shown in Shi and $\mathrm{Qu}(2017)$.

However, if $\operatorname{corr}\left(x_{i j}, x_{i k}\right) \neq 0$ for some $k, k \neq j$, then

$$
P_{d, j}^{*}=\Phi\left(\frac{\beta_{j 0}-\sqrt{\lambda} \sigma}{\sigma /\left[\sqrt{n} \sqrt{\left.\{\operatorname{corr}(\tilde{\mathbf{X}})\}_{j+1, j+1}^{-1}\right]}\right]}\right)+\Phi\left(\frac{-\beta_{j 0}-\sqrt{\lambda} \sigma}{\sigma /\left[\sqrt{n} \sqrt{\{\operatorname{corr}(\tilde{\mathbf{X}})\}_{j+1, j+1}^{-1}}\right]}\right) .
$$

Thus, $P_{d, j}^{*}$ also depends on correlations among covariates. Given any values in $P_{d, j}^{*}$ except 


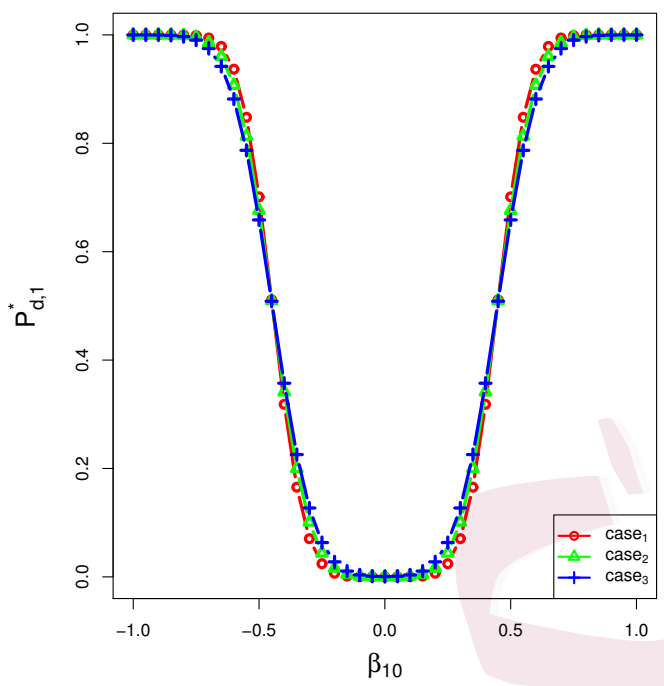

Figure 1: The plots for $P_{d, 1}^{*}$ as $\beta_{10}$ varies under three different cases in linear regression models. In case 1 , the correlation structure of $\mathbf{X}$ is taken to be the independence correlation structure; in case 2 , the correlation structure of $\mathbf{X}$ is taken to be the $\mathrm{AR}(1)$ correlation structure with $\rho=0.5$; in case 3 , the correlation structure of $\mathbf{X}$ is taken to be the exchangeable correlation structure with $\rho=0.5$. In all cases, $n=100, p=5, \lambda=0.2, \sigma=1$, and $\beta_{10}$ varies between -1 and 1 with a step size of 0.05 .

$\beta_{j 0}, P_{d, j}^{*}$ is still a symmetric function of $\beta_{j 0}$ and an increasing function of $\left|\beta_{j 0}\right|$. However, under different correlation structures of $\tilde{\mathbf{X}}, P_{d, j}^{*}$ can have different shapes as the value of $\beta_{j 0}$ varies. Therefore, both the value of $\left|\beta_{j 0}\right|$ and the correlation structure of $\tilde{\mathbf{X}}$ influence the signal strength of $\boldsymbol{X}_{j}$, which are illustrated in Figure 1 .

\section{Case Two: Logistic regression model}

Under the logistic regression model setting,

$$
\mathrm{E}\left(y_{i} \mid \mathbf{x}_{i}\right)=p_{i}=\frac{\exp \left(\alpha_{0}+\mathbf{x}_{i}^{\top} \boldsymbol{\beta}_{0}\right)}{1+\exp \left(\alpha_{0}+\mathbf{x}_{i}^{\top} \boldsymbol{\beta}_{0}\right)}
$$

We can obtain that in (3.1), $D_{0, i i}=p_{i}\left(1-p_{i}\right)$ and $\mathbf{D}_{0}=\operatorname{diag}\left\{p_{1}\left(1-p_{1}\right), \ldots, p_{n}\left(1-p_{n}\right)\right\}$. Thus, $P_{d, j}^{*}$ not only depends on $\beta_{j 0}$, but also depends on $\gamma_{0}^{-j}$, the coefficients of other covariates. This is a fundamental difference between logistic regression models and linear 
regression models in terms of selection probability. In contrast to linear regression models, $\mathbf{x}_{i}$ influences $P_{d, j}^{*}$ through the matrix $\mathrm{E}\left[\widetilde{\mathbf{X}}^{\top} \operatorname{diag}\left\{p_{1}\left(1-p_{1}\right), \ldots, p_{n}\left(1-p_{n}\right)\right\} \widetilde{\mathbf{X}}\right]$ rather than the correlation matrix of $\tilde{\mathbf{X}}$ in logistic regression models. In addition, in Supplementary Material S2.1, we show that $P_{d, j}^{*}$ is not necessarily a symmetric function of $\beta_{j 0}$ given other values in $P_{d, j}^{*}$. Thus, $\left|\beta_{j 0}\right|$ cannot be used to measure the signal strength of $\boldsymbol{X}_{j}$ instead of $P_{d, j}^{*}$, which is different from that in Shi and Qu (2017).

In addition, for the logistic regression model, the range of $\gamma_{0}$ is bounded so that $p_{i}$ can satisfy the condition of $0<c_{1}<p_{i}<c_{2}<1$, where $c_{1}$ and $c_{2}$ are some positive constants. We show that given any values in $P_{d, j}^{*}$ except $\beta_{j 0}, P_{d, j}^{*}$ is an increasing function of $\beta_{j 0}$ if $0<\beta_{j 0}<c_{3}$ and $P_{d, j}^{*}$ is a decreasing function of $\beta_{j 0}$ if $-c_{4}<\beta_{j 0}<0$, where $c_{3}$ and $c_{4}$ are some bounded positive constants depending on $c_{1}$ and $c_{2}$. Proofs of the above findings are provided in Supplementary Material S2.2. We also illustrate these properties by Figure 2 . Note that in this case, the response variable has two categories, it can be easily extended to the case where the response variable has more than two categories. 


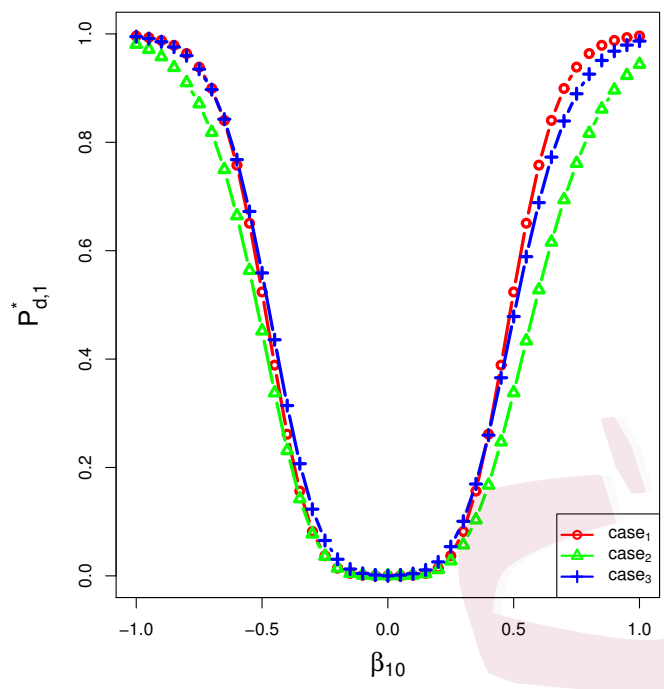

Figure 2: The plots for $P_{d, 1}^{*}$ as $\beta_{10}$ varies under three different cases in logistic regression models. In case 1, $\boldsymbol{X}_{1}$ and $\boldsymbol{X}_{2}$ both follow the standard normal distribution, and $\boldsymbol{X}_{1}$ and $\boldsymbol{X}_{2}$ are independent; in case $2, \boldsymbol{X}_{1}$ and $\boldsymbol{X}_{2}$ both follow the centralized exponential distribution with mean 0 and variance 1, and $\boldsymbol{X}_{1}$ and $\boldsymbol{X}_{2}$ are independent; in case $3, \boldsymbol{X}_{1}$ and $\boldsymbol{X}_{2}$ both follow the standard normal distribution, and $\boldsymbol{X}_{1}$ and $\boldsymbol{X}_{2}$ have the correlation of 0.5. In all cases, $n=300, \gamma_{0}=\left(0.3, \beta_{10}, 0.2\right)^{\prime}, \lambda=0.05$, $\beta_{10}$ varies between -1 and 1 with a step size of 0.05 .

\section{Case Three: Poisson regression model}

Under the Poisson regression model setting,

$$
P\left(y_{i}=y \mid \mathbf{x}_{i}\right)=\frac{\lambda_{i}^{y}}{y !} \exp \left(-\lambda_{i}\right)
$$

where $\lambda_{i}=\mathrm{E}\left(y_{i} \mid \mathbf{x}_{i}\right)=\exp \left(\alpha_{0}+\mathbf{x}_{i}^{\top} \boldsymbol{\beta}_{0}\right)$. Then in (3.1), $D_{0, i i}=\lambda_{i}$ and $\mathbf{D}_{0}=\operatorname{diag}\left\{\lambda_{1}, \ldots, \lambda_{n}\right\}$.

We obtain similar conclusions as those for logistic regression models, except that $P_{d, j}^{*}$ is influenced by $\mathbf{x}_{i}$ through the matrix $\mathrm{E}\left[\widetilde{\mathbf{X}}^{\top} \operatorname{diag}\left\{\lambda_{1}, \ldots, \lambda_{n}\right\} \widetilde{\mathbf{X}}\right]$. Note that under Assumption 1 , the range of $\gamma_{0}$ is bounded. Given any other values in $P_{d, j}^{*}$ except $\beta_{j 0}, P_{d, j}^{*}$ is an increasing function of $\beta_{j 0}$ if $0<\beta_{j 0}<c_{5}$ and $P_{d, j}^{*}$ is a decreasing function of $\beta_{j 0}$ if $-c_{6}<\beta_{j 0}<0$, where $c_{5}$ and $c_{6}$ are some bounded positive constants. The proof for this finding is provided in Supplementary Material S2.2. Figure 3 provides an illustration of $P_{d, j}^{*}$. 


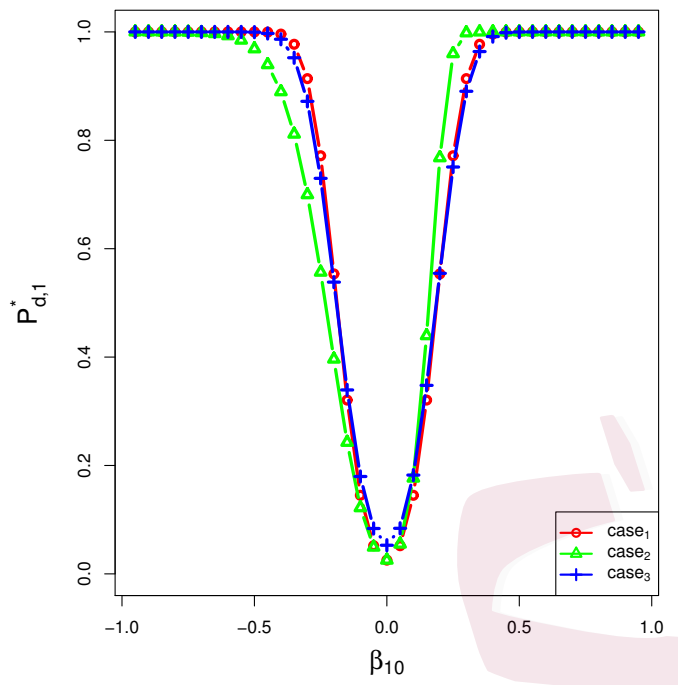

Figure 3: The plots for $P_{d, 1}^{*}$ as $\beta_{10}$ varies under three different cases in Poisson regression models. In case 1, $\boldsymbol{X}_{1}$ and $\boldsymbol{X}_{2}$ both follow the standard normal distribution, and $\boldsymbol{X}_{1}$ and $\boldsymbol{X}_{2}$ are independent; in case $2, \boldsymbol{X}_{1}$ and $\boldsymbol{X}_{2}$ both follow the centralized exponential distribution with mean 0 and variance 1, and $\boldsymbol{X}_{1}$ and $\boldsymbol{X}_{2}$ are independent; in case $3, \boldsymbol{X}_{1}$ and $\boldsymbol{X}_{2}$ both follow the standard normal distribution, and $\boldsymbol{X}_{1}$ and $\boldsymbol{X}_{2}$ have the correlation of 0.5. In all cases, $n=300, \gamma_{0}=\left(0.3, \beta_{10}, 0.2\right)^{\prime}, \lambda=0.05$, $\beta_{10}$ varies between -0.95 and 0.95 with a step size of 0.05 .

Finite sample properties of $P_{d, j}^{*}$ under other likelihood-based models can be analyzed similarly. In general, $P_{d, j}^{*}$ is an integrated indicator. It shows how the selection probability of $\boldsymbol{X}_{j}$ is influenced by $\boldsymbol{\gamma}_{0}, \mathbf{x}_{i}, n$ and $\lambda$ in finite samples. Given other values in $P_{d, j}^{*}$ except $\beta_{j 0}, P_{d, j}^{*}$ is not necessarily a symmetric function of $\beta_{j 0}$ or an increasing function of $\left|\beta_{j 0}\right|$.

Based on the above analysis, we propose to utilize $P_{d, j}^{*}$ to measure the signal strength levels directly, rather than utilizing $\left|\beta_{j 0}\right|$. Intuitively, if $P_{d, j}^{*}$ is close to one, then the variable $\boldsymbol{X}_{j}$ is defined to be a strong signal; if $P_{d, j}^{*}$ is close to zero, then the variable $\boldsymbol{X}_{j}$ is defined to be a noise variable; if $P_{d, j}^{*}$ is in-between the strong and noise levels, then the variable $\boldsymbol{X}_{j}$ is defined to be a weak signal. Specifically, we introduce two threshold values, $\delta^{s}$ and $\delta^{w}$, then 
the three levels of signal strength can be defined as

$$
\left\{\begin{array}{l}
\boldsymbol{X}_{j} \in \mathbf{X}^{(S)}, \quad \text { if } \quad P_{d, j}^{*}>\delta^{s} ; \\
\boldsymbol{X}_{j} \in \mathbf{X}^{(W)}, \quad \text { if } \quad \delta^{w}<P_{d, j}^{*} \leq \delta^{s} ; \\
\boldsymbol{X}_{j} \in \mathbf{X}^{(N)}, \quad \text { if } \quad P_{d, j}^{*} \leq \delta^{w},
\end{array}\right.
$$

where $0<\tau^{w} \leq \delta^{w}<\delta^{s} \leq \tau^{s} \leq 1, \tau^{w}=\min _{j} P_{d, j}^{*}$ and $\tau^{s}=\max _{j} P_{d, j}^{*}$. Obviously, a stronger signal is easier to be selected through the variable selection process than a weaker signal.

\subsection{Weak signal identification}

In this section, we provide details on identifying weak signals. Based on the analysis in Section 3.1. the approximated selection probability $P_{d, j}^{*}$ depends on the true parameter $\gamma_{0}$ and the distribution of $\mathbf{x}_{i}$, but they are always unknown in practice. In the following, we estimate $P_{d, j}^{*}$ by plugging in the maximum likelihood estimator $\gamma^{(0)}$ and the empirical mean of random variables in 3.1 . That is,

$$
\begin{array}{r}
\hat{P}_{d, j}^{*}=\Phi\left(\begin{array}{c}
\left.\frac{-\sqrt{\frac{n \lambda \sum_{i=1}^{n} D_{i i}^{(0)}}{\sum_{i=1}^{n} D_{i i}^{(0)} x_{i j}^{2} \sum_{i=1}^{n} D_{i i}^{(0)}-\left(\sum_{i=1}^{n} D_{i i}^{(0)} x_{i j}\right)^{2}}}+\beta_{j}^{(0)}}{\sqrt{\left(\widetilde{\mathbf{X}}^{\top} \mathbf{D}^{(0)} \widetilde{\mathbf{X}}\right)_{j+1, j+1}^{-1}}}\right) \\
+\Phi\left(\frac{-\sqrt{\frac{n \lambda \sum_{i=1}^{n} D_{i i}^{(0)}}{\sum_{i=1}^{n} D_{i i}^{(0)} x_{i j}^{2} \sum_{i=1}^{n} D_{i i}^{(0)}-\left(\sum_{i=1}^{n} D_{i i}^{(0)} x_{i j}\right)^{2}}}-\beta_{j}^{(0)}}{\sqrt{\left(\widetilde{\mathbf{X}}^{\top} \mathbf{D}^{(0)} \widetilde{\mathbf{X}}\right)_{j+1, j+1}^{-1}}}\right) .
\end{array}\right. \\
\\
+\Phi
\end{array}
$$

In practice, we identify the signal strength level of $\boldsymbol{X}_{j}$ based on $\hat{P}_{d, j}^{*}$ and introduce two threshold values $\delta_{1}$ and $\delta_{2}$. We denote the identified subsets of strong signals, weak signals 
and noise variables as $\hat{\mathbf{S}}^{(S)}, \hat{\mathbf{S}}^{(W)}$ and $\hat{\mathbf{S}}^{(N)}$, respectively, and they are

$$
\left\{\begin{array}{l}
\hat{\mathbf{S}}^{(S)}=\left\{j: \hat{P}_{d, j}^{*}>\delta_{1}\right\} ; \\
\hat{\mathbf{S}}^{(W)}=\left\{j: \delta_{2}<\hat{P}_{d, j}^{*} \leq \delta_{1}\right\} \\
\hat{\mathbf{S}}^{(N)}=\left\{j: \hat{P}_{d, j}^{*} \leq \delta_{2}\right\} .
\end{array}\right.
$$

The selections of $\delta_{1}$ and $\delta_{2}$ are crucial for determining the signal type. The threshold value $\delta_{1}$ is selected to ensure that we can identify strong signals when the selection probabilities of signals are high. Assume $\alpha$ is a significance level, and we choose $\delta_{1}$ to be larger than $1-\alpha$ so that the identified strong signals are strong. The threshold value $\delta_{2}$ is selected to control the false positive rate of selecting variable $\boldsymbol{X}_{j}$. Denote the false positive rate as $\tau$, then $\tau$ can be defined as

$$
\tau=P\left(j \notin \hat{\mathbf{S}}^{(N)} \mid \beta_{j 0}=0, \gamma_{0}^{-j}\right)=P\left(\hat{P}_{d, j}^{*}>\delta_{2} \mid \beta_{j 0}=0, \gamma_{0}^{-j}\right) .
$$

Thus, $\delta_{2}$ can be estimated based on (3.5). Since the value of $\gamma_{0}$ is unknown in practice, we estimate it using the one-step adaptive Lasso estimator $\gamma^{(1)}$. Furthermore, to make the estimated value of false positive rate equal $\tau$ based on the observed data, we take the value of $\delta_{2}$ as the $100(1-\tau) \%$ quantile of $\left\{\hat{P}_{d, j}^{*}: \beta_{j}^{(1)}=0, j=1, \ldots, p\right\}$. Since we intend to recover weak signals given finite samples, $\tau$ is chosen to be larger than zero. However, the value of $\tau$ cannot be too large as there is a trade-off between recovering weak signals and including noise variables. In practice, if we want to recover more weak signals, we can choose a larger $\tau$; if we want to make the false positive rate lower, we can choose a smaller $\tau$. In the simulation studies, we make a sensitivity analysis of the choice of $\delta_{1}$ and $\tau$. 


\section{Weak signal inference}

In this section, we propose a two-step inference procedure for the construction of confidence intervals for regression coefficients. The two-step inference procedure consists of two parts: if a covariate is identified as a strong signal, then its confidence interval will be constructed based on the asymptotic theory for the non-zero one-step adaptive Lasso estimator (Zou and Li, 2008); and if a covariate is identified as a weak signal or a noise variable, then we provide a confidence interval based on the following inference theory for the maximum likelihood estimator.

Similar to the theory in Zou and Li (2008), we can obtain the asymptotic distribution of the one-step adaptive Lasso estimator. Without loss of generality, assume $\mathscr{A}_{n}=\{1, \ldots, s\}$, where $s$ is the number of non-zero elements in $\boldsymbol{\beta}^{(1)}$. Define $\mathscr{B}_{n}=\left\{k: \gamma_{k}^{(1)} \neq 0, k=\right.$ $1, \ldots, p+1\}$, then $\mathscr{B}_{n}=\{1, \ldots, s+1\}$. Although the one-step adaptive Lasso estimator $\boldsymbol{\beta}_{\mathscr{Q}_{n}}^{(1)}$ is biased due to the shrinkage effect in finite samples, we can construct a de-biased confidence interval for the true coefficient based on the estimated bias and covariance matrix for $\boldsymbol{\beta}_{\mathscr{A}_{n}}^{(1)}$, as shown in Theorem 1 . Proof of Theorem 1 is given in Supplementary Material S3.

Theorem 1. Denote $\mathbf{X}^{\top} \mathbf{D}^{\dagger(0)} \mathbf{X}$ and $\widetilde{\mathbf{X}}^{\top} \mathbf{D}^{(0)} \widetilde{\mathbf{X}} / n$ as $\mathbf{Z}^{(0)}$ and $\mathbf{I}^{(0)}$, respectively. The estimators of bias and covariance matrix of $\boldsymbol{\beta}_{\mathscr{A}_{n}}^{(1)}$ are given by

$$
\widehat{\operatorname{bias}}\left(\boldsymbol{\beta}_{\mathscr{\mathscr { A } _ { n }}}^{(1)}\right)=-\left\{\frac{1}{n} \mathbf{Z}_{\mathscr{A}_{n}}^{(0)}+\Sigma_{\lambda}\left(\boldsymbol{\beta}_{\mathscr{A}}^{(0)}, \boldsymbol{\beta}_{\mathscr{A}_{n}}^{(1)}\right)\right\}^{-1}\left(\frac{\lambda}{\left|\beta_{1}^{(0)}\right|} \operatorname{sgn}\left(\beta_{1}^{(1)}\right), \ldots, \frac{\lambda}{\left|\beta_{s}^{(0)}\right|} \operatorname{sgn}\left(\beta_{s}^{(1)}\right)\right)^{\top}
$$


and

$$
\begin{aligned}
\widehat{\operatorname{cov}}\left(\boldsymbol{\beta}_{\mathscr{A}_{n}}^{(1)}\right)=\frac{1}{n^{3}}\left\{\frac{1}{n} \mathbf{Z}_{\mathscr{A}_{n}}^{(0)}+\Sigma_{\lambda}\left(\boldsymbol{\beta}_{\mathscr{A}_{n}}^{(0)}, \boldsymbol{\beta}_{\mathscr{A}_{n}}^{(1)}\right)\right\}^{-1} \mathbf{Z}_{\mathscr{A}_{n}}^{(0)}\left\{\left(\mathbf{I}_{\mathscr{B}_{n}}^{(0)}\right)^{-1}\right\} \mathscr{\mathscr { A }}_{n} \mathbf{Z}_{\mathscr{A}_{n}}^{(0)} \\
\times\left\{\frac{1}{n} \mathbf{Z}_{\mathscr{A}_{n}}^{(0)}+\Sigma_{\lambda}\left(\boldsymbol{\beta}_{\mathscr{A}_{n}}^{(0)}, \boldsymbol{\beta}_{\mathscr{A}_{n}}^{(1)}\right)\right\}^{-1},
\end{aligned}
$$

where $\Sigma_{\lambda}\left(\boldsymbol{\beta}_{\mathscr{A}_{n}}^{(0)}, \boldsymbol{\beta}_{\mathscr{A}_{n}}^{(1)}\right)=\operatorname{diag}\left\{\lambda /\left(\left|\beta_{1}^{(0)}\right|\left|\beta_{1}^{(1)}\right|\right), \ldots, \lambda /\left(\left|\beta_{s}^{(0)}\right|\left|\beta_{s}^{(1)}\right|\right)\right\}, \mathbf{Z}_{\mathscr{A}_{n}}^{(0)}$ is the sub-matrix of $\mathbf{Z}^{(0)}$ corresponding to $\boldsymbol{\beta}_{\mathscr{A}_{n}}^{(0)}$, and $\mathbf{I}_{\mathscr{B}_{n}}^{(0)}$ is the sub-matrix of $\mathbf{I}^{(0)}$ corresponding to $\boldsymbol{\gamma}_{\mathscr{B}_{n}}^{(0)}$.

Based on Theorem 1 , if the covariate $\boldsymbol{X}_{j}$ is identified as a strong signal, then the $100(1-$ $\alpha) \%$ confidence interval for $\beta_{j 0}$ can be constructed as

$$
\left(\beta_{j}^{(1)}-\hat{b}_{j}-z_{\alpha / 2} \hat{\sigma}_{j}, \beta_{j}^{(1)}-\hat{b}_{j}+z_{\alpha / 2} \hat{\sigma}_{j}\right)
$$

where $\hat{b}_{j}$ is the corresponding component of $\widehat{\operatorname{bias}}\left(\boldsymbol{\beta}_{\mathscr{A}_{n}}^{(1)}\right)$ and $\hat{\sigma}_{j}$ is the square root of the corresponding diagonal component of $\widehat{\operatorname{cov}}\left(\boldsymbol{\beta}_{\mathscr{A}_{n}}^{(1)}\right)$.

If the covariate $\boldsymbol{X}_{j}$ is identified as a weak signal or a noise variable, then the $100(1-\alpha) \%$ confidence interval for $\beta_{j 0}$ can be constructed as

$$
\left(\beta_{j}^{(0)}-z_{\alpha / 2} \sigma_{j}^{(0)}, \beta_{j}^{(0)}+z_{\alpha / 2} \sigma_{j}^{(0)}\right)
$$

where $\sigma_{j}^{(0)}$ is the square root of the corresponding diagonal component of $\widehat{\operatorname{cov}}\left(\boldsymbol{\gamma}^{(0)}\right)=$ $\left(\widetilde{\mathbf{X}}^{\top} \mathbf{D}^{(0)} \widetilde{\mathbf{X}}\right)^{-1}$

Remark 1. Note that in Shi and Qu (2017), they did not construct confidence intervals for noise variables. In contrast to Shi and $\mathrm{Qu}(2017)$, we also construct confidence intervals for noise variables. As shown in Figure 6 in the simulation studies, this improves coverage 
probabilities for noise variables and weak signals. Using the two-step inference method based on the idea of Shi and $\mathrm{Qu}(2017)$, the coverage probabilities for noise variables tend to be lower than $1-\alpha$ and the coverage probabilities for weak signals tend to be higher than $1-\alpha$. This is because for noise variables, we construct confidence intervals for them only when they are misidentified as weak signals or strong signals, in which case the estimated values of coefficients tend to be far away from the true values, leading to lower coverage probabilities. For weak signals, we do not construct confidence intervals for them when they are misidentified as noise variables, making the coverage probabilities of confidence intervals higher. To solve these problems, we propose to construct confidence intervals for the identified noise variables as well. As a result, the coverage probabilities of confidence intervals become closer to $1-\alpha$.

In summary, our proposed confidence interval for $\beta_{j 0}$ can be written as

$$
\left(\beta_{j}^{(1)}-\hat{b}_{j}-z_{\alpha / 2} \hat{\sigma}_{j}, \beta_{j}^{(1)}-\hat{b}_{j}+z_{\alpha / 2} \hat{\sigma}_{j}\right) \mathrm{I}\left\{j \in \hat{\mathbf{S}}^{(S)}\right\}
$$

$$
+\left(\beta_{j}^{(0)}-z_{\alpha / 2} \sigma_{j}^{(0)}, \beta_{j}^{(0)}+z_{\alpha / 2} \sigma_{j}^{(0)}\right) \mathrm{I}\left\{j \in \hat{\mathbf{S}}^{(W)} \cup \hat{\mathbf{S}}^{(N)}\right\},
$$

which combines both 4.1) and 4.2.

\section{Simulation studies}

In this section, we conduct simulation studies to evaluate the finite sample performance of the proposed signal identification criterion and the two-step inference procedure. Consider 
the following logistic regression model:

$$
P\left(y_{i}=1 \mid \mathbf{x}_{i}\right)=\frac{\exp \left(\alpha_{0}+\mathbf{x}_{i}^{\top} \boldsymbol{\beta}_{0}\right)}{1+\exp \left(\alpha_{0}+\mathbf{x}_{i}^{\top} \boldsymbol{\beta}_{0}\right)}, \quad i=1, \ldots, n .
$$

We generate the covariate vector $\mathbf{x}_{i}=\left(x_{i 1}, \ldots, x_{i p}\right)^{\top}$ from a multivariate normal distribution with mean zero and covariance matrix $\mathbf{R}(\rho) \sigma^{2}$, where $\mathbf{R}(\rho)$ is a correlation matrix with the $\mathrm{AR}(1)$ correlation structure and $\sigma^{2}=1$. All the generated covariates are standardized by subtracting their sample means and divided by their sample standard deviations, separately. For each setting, we choose $n=350$ or $550, p=25$ or $35, \rho=0,0.2$ or 0.5 and $\alpha_{0}=0.5$. The regression coefficient vector $\boldsymbol{\beta}_{0}$ is set to be $(1,1,0.5, \theta, \underbrace{0, \ldots, 0}_{p-4})^{\top}$, which consists of two large coefficients 1 , one moderate size coefficient 0.5 , one varying coefficient $\theta$ and $(p-4)$ zero coefficients. The coefficient $\theta$ ranges from 0 to 1 with a step size of 0.05 . In each simulation setting, we repeat simulations for 500 times. The implementation details of the one-step adaptive Lasso estimators are given in Supplementary Material S4.

Figure 4 displays the results for different types of selection probability for $\boldsymbol{X}_{4}$ when $\rho=0$. In Figure 4, the approximated selection probability based on (3.1) is close to the empirical selection probability, indicating a small approximation error from the approximated selection probability. In addition, both the empirical selection probability and the approximated selection probability increase with $\theta$, implying that a larger value of $\theta$ leads to a stronger signal strength. This observation supports the result in Section 3.1. Although the median of the estimated selection probabilities is not too close to the empirical selection probability when $\theta$ is small, the estimated selection probability still increases with the signal strength. We can still use the estimated selection probability to identify the signal strength level. The simulation results for correlated covariates are provided in Figures S1 and S2 
of Supplementary Material S5, and the approximated selection probability is similar to the empirical selection probability. In addition, the empirical selection probability, the approximated selection probability, and the estimated selection probability in general increase with the value of $\theta$. Thus, we can also identify the signal strength level based on the value of $\theta$.

We then identify whether a covariate is a strong signal, weak signal or noise variable based on the criterion in (3.4). For illustration, we choose $\delta_{1}$ to be 0.99 and $\tau$ to be 0.1 . Figure 5 represents the empirical probabilities of assigning the covariate $\boldsymbol{X}_{4}$ to different signal categories as $\theta$ varies and $\rho=0$. Figure 5 shows that when $\theta$ is close to zero, $\boldsymbol{X}_{4}$ is more likely to be identified as a noise variable; when $\theta$ is far away from zero and one, the empirical probability of $\boldsymbol{X}_{4}$ being identified as a weak signal is highest; as $\theta$ becomes larger, the empirical probability of $\boldsymbol{X}_{4}$ being identified as a strong signal becomes more dominant and gradually increases to one. The results for correlated covariates are given in Figures S3 and S4 of Supplementary Material S5, and we have similar findings. Therefore, our proposed signal identification criterion (3.4) performs well in practice.

After identifying the signal strength levels, we construct the $95 \%$ confidence intervals based on the proposed two-step inference procedure. We also compare our method with the two-step inference method based on the idea of Shi and Qu (2017), which does not construct confidence intervals for identified noise variables. In addition, we construct confidence intervals based on the asymptotic theory for the one-step adaptive Lasso estimator as shown in (4.1), the maximum likelihood estimation method as shown in 4.2 , the perturbation method (Minnier et al., 2011), the estimating equation-based method (Neykov et al., 2018), the standard bootstrap method (Efron and Tibshirani, 1994), the smoothed bootstrap method (Efron, 2014), the de-biased Lasso method (Javanmard and Montanari, 

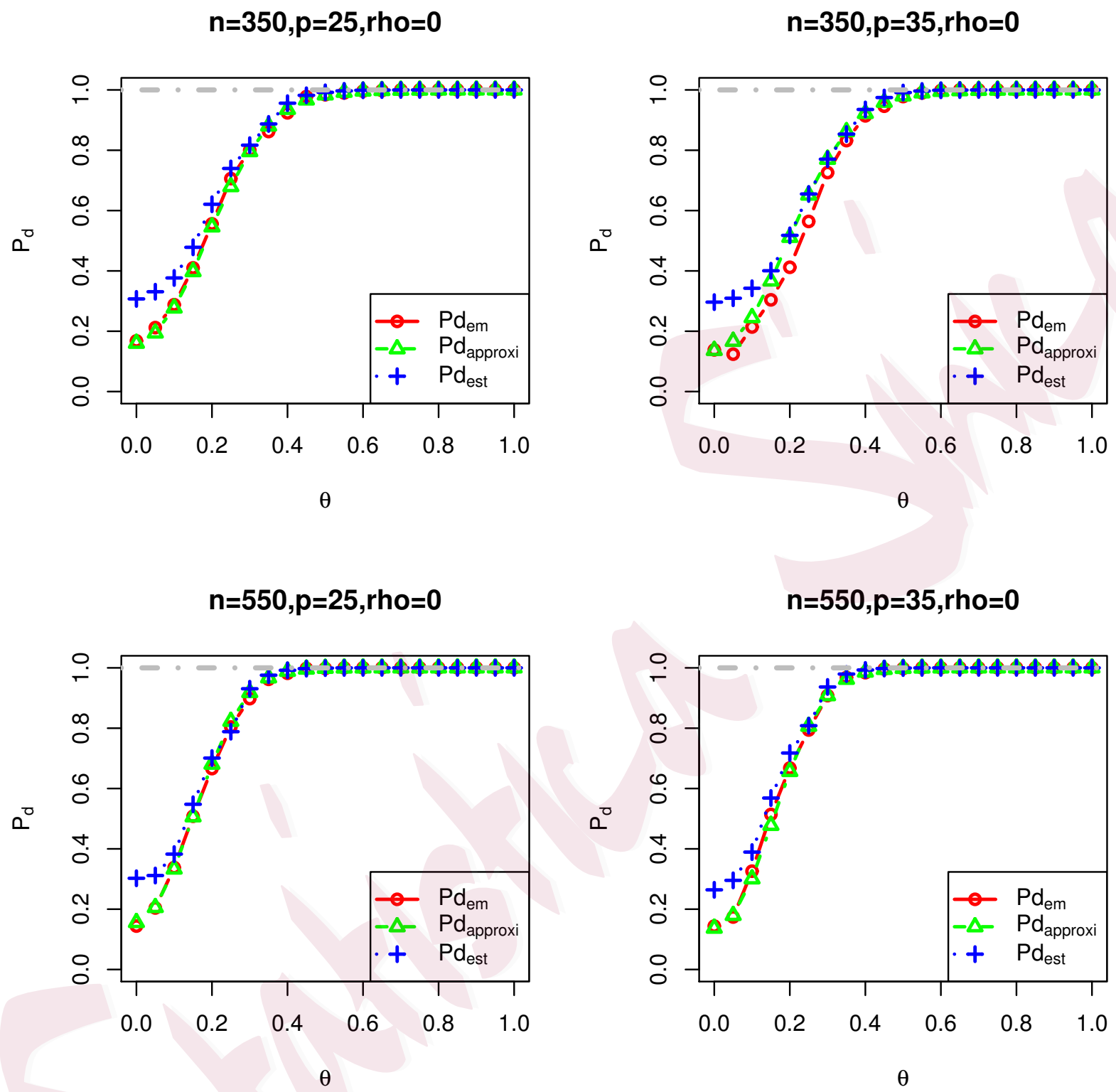

Figure 4: Different types of selection probability for $\boldsymbol{X}_{4}$ when $\rho=0 . \operatorname{Pd}_{\mathrm{em}}$ : empirical selection probability, which equals the empirical probability of $\left\{\theta^{(1)} \neq 0\right\}$ based on 500 Monte-Carlo samples; $\mathrm{Pd}_{\text {approxi: }}$ approximated selection probability based on (3.1), where the expectations in (3.1) are calculated by using the function cubintegrate in $\mathrm{R}$; $\mathrm{Pd}_{\text {est }}$ : median of estimated selection probabilities based on (3.3) for 500 Monte-Carlo samples.

2014; Van de Geer et al., 2014; Zhang and Zhang, 2014) and two different types of bootstrap

de-biased Lasso methods (Dezeure et al., 2017). The number of bootstrap resampling is set to be 4000 for all the bootstrap methods, and the resampling number is set to be 500 

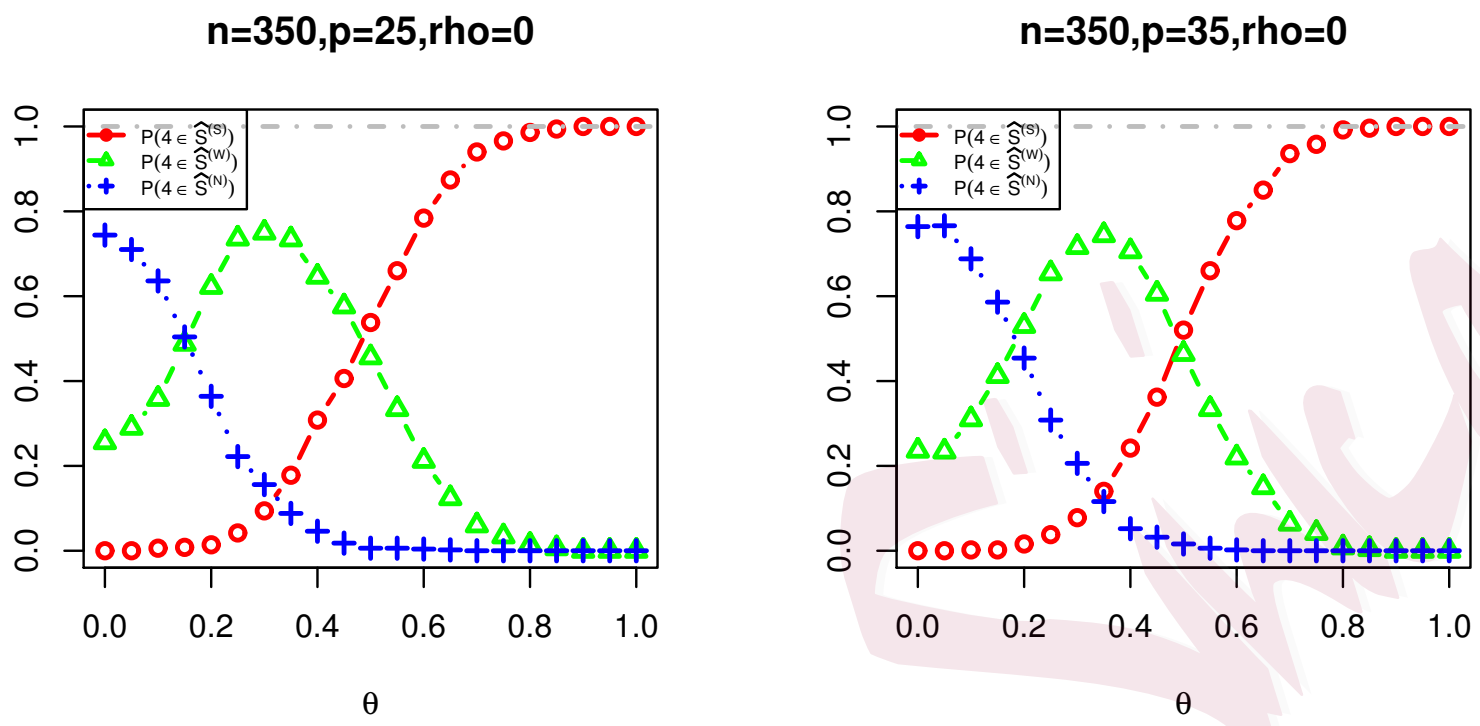

$\mathrm{n}=550, \mathrm{p}=25, \mathrm{rho}=0$

$\mathrm{n}=550, \mathrm{p}=35, \mathrm{rho}=0$

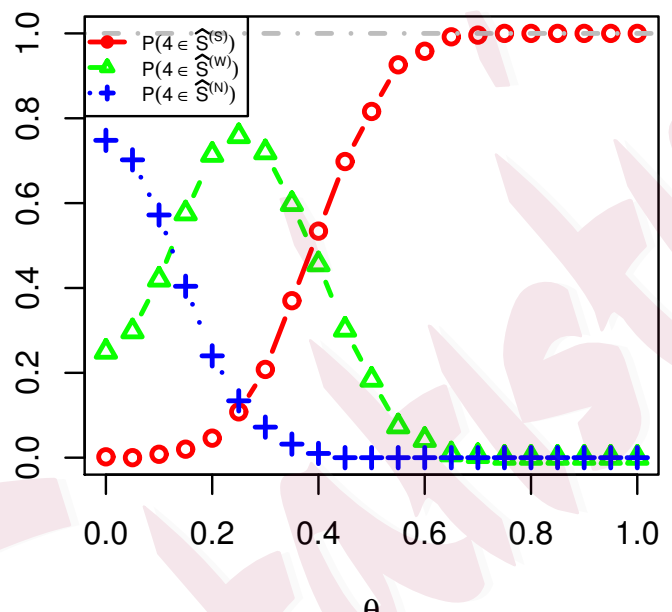

$\theta$

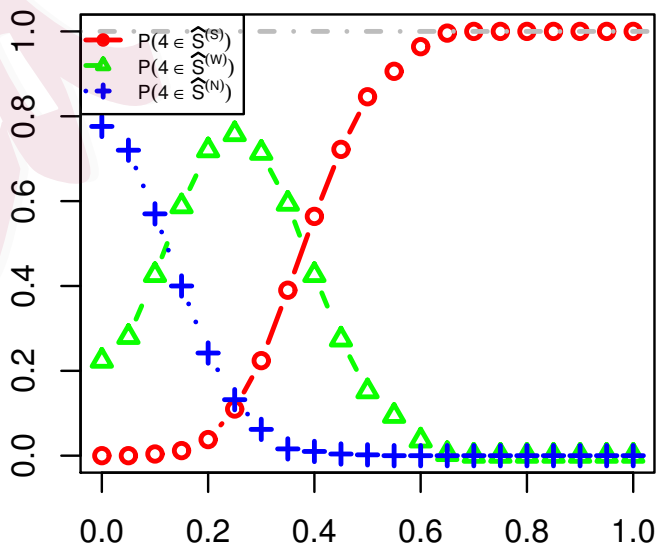

Figure 5: Empirical probabilities of assigning the covariate $\boldsymbol{X}_{4}$ to different signal categories when $\rho=0$.

for the perturbation method. The implementation details of the estimating equation-based method and two types of bootstrap de-biased Lasso methods can be found in Supplementary Material S4. For the method based on the asymptotic theory for the one-step adaptive Lasso estimator, if a variable is not selected, then we do not construct a confidence interval for it 
as the asymptotic normality is established only for the selected variables.

Figures 6 and 7 provide coverage probabilities of the $95 \%$ confidence intervals as $\theta$ varies and $(n, p, \rho)=(350,25,0)$. In Figures 6 and 7 , the vertical line on the left shows whether $\boldsymbol{X}_{4}$ is more likely to be identified as a noise variable or weak signal, and the vertical line on the right distinguishes whether $\boldsymbol{X}_{4}$ is more likely to be identified as a weak signal or strong signal. The threshold values are obtained from Figure 5. Comparing the proposed two-step inference method with the two-step inference method based on the idea of Shi and $\mathrm{Qu}$ (2017), when $\theta$ is small, the proposed two-step inference method performs much better than the two-step inference method based on the idea of Shi and Qu (2017). When $\theta$ is close to zero, the coverage probability of the asymptotic method is too low and close to zero, while the perturbation method, standard bootstrap method, smoothed bootstrap method, and type-I bootstrap de-biased Lasso method provide over-coverage confidence intervals with coverage probabilities approximating to one. When the signal is weak, the asymptotic method, perturbation method, standard bootstrap method, smoothed bootstrap method and type-I bootstrap de-biased Lasso method all perform poorly whereas their coverage probabilities are much lower than 95\%. In addition, the coverage probability of the estimating equation-based method is slightly lower than $95 \%$. When the signal is stronger, the performance of the maximum likelihood estimation method, estimating equation-based method, de-biased Lasso method, and type-I bootstrap de-biased Lasso method also become worse. However, the coverage probabilities of the $95 \%$ confidence intervals for the proposed method and type-II bootstrap de-biased Lasso method are close to $95 \%$ under all signal strength levels of $\theta$.

Figure 8 provides average widths of the $95 \%$ confidence intervals as $\theta$ varies and $(n, p, \rho)=$ 


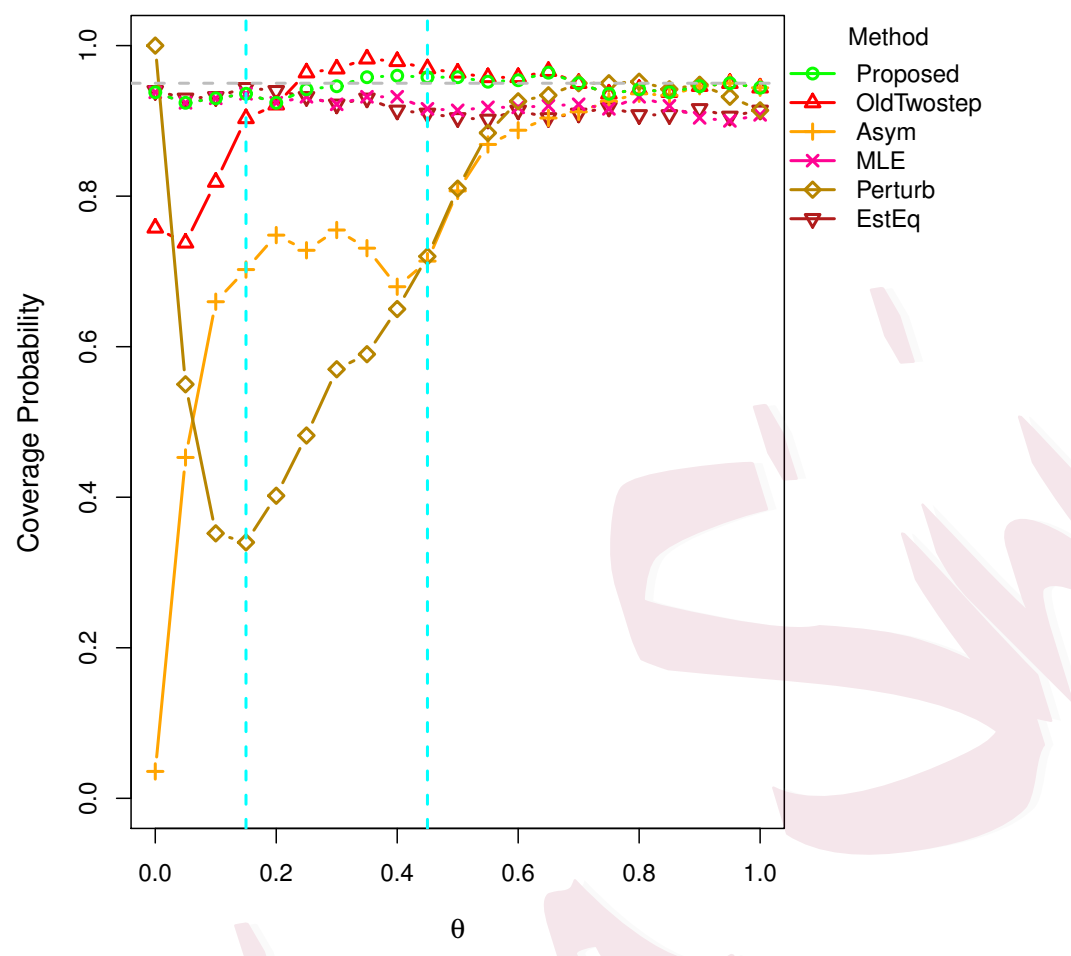

Figure 6: Coverage probabilities of the $95 \%$ confidence intervals when $(n, p, \rho)=(350,25,0)$. Proposed: the proposed two-step inference method; OldTwostep: the two-step inference method based on the idea of Shi and Qu (2017), which does not construct confidence intervals for identified noise variables; Asym: the method based on the asymptotic theory using the one-step adaptive Lasso estimator; MLE: the maximum likelihood estimation method; Perturb: the perturbation method; EstEq: the estimating equation-based method.

$(350,25,0)$. Note that the widths of confidence intervals for the two types of two-step inference methods are both very close, while their coverage probabilities are not similar when $\theta$ is small. The width of confidence interval using the proposed method is between those using the maximum likelihood estimation method and the asymptotic method. This is not surprising as the proposed method combines the strengths of the maximum likelihood estimation method and the asymptotic method. Although the confidence intervals based on the asymptotic method, perturbation method, standard bootstrap method, and smoothed bootstrap method are narrow when $\theta$ is close to zero, the coverage probabilities are not accurate since they are either too small or too large. When the signal is strong, the widths of 


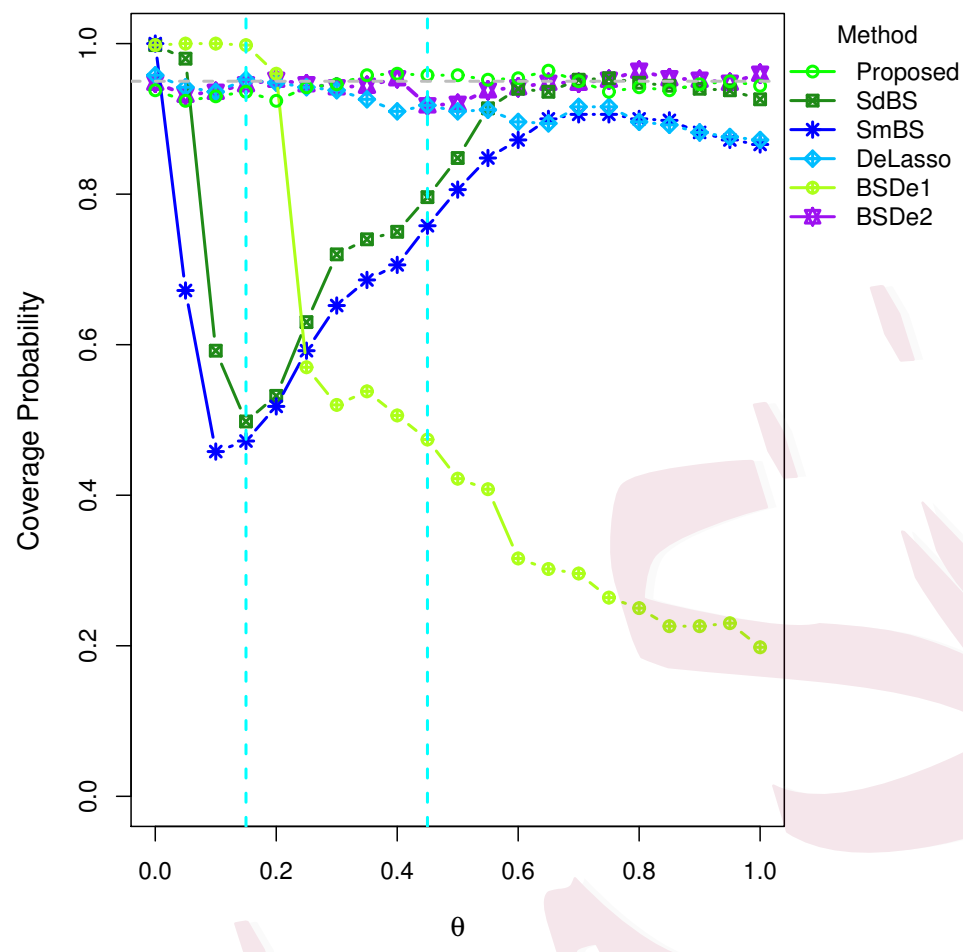

Figure 7: Coverage probabilities of the $95 \%$ confidence intervals when $(n, p, \rho)=(350,25,0)$. Proposed: the proposed two-step inference method; SdBS: the standard bootstrap method; SmBS: the smoothed bootstrap method; DeLasso: the de-biased Lasso method; BSDe1: the type-I bootstrap de-biased Lasso method; BSDe2: the type-II bootstrap de-biased Lasso method.

confidence intervals for the perturbation method, standard bootstrap method, and smoothed bootstrap method are in general larger than that for the proposed method. Although the estimating equation-based method, de-biased Lasso method, and type-I bootstrap de-biased Lasso method have shorter confidence intervals than the proposed method, their coverage probabilities of confidence intervals are lower as the signal becomes stronger. Overall, the confidence interval for the type-II bootstrap de-biased Lasso method is wider than that for the proposed method.

The coverage probabilities and average widths of the $95 \%$ confidence intervals under all simulation settings are summarized in Tables S1-S4 of Supplementary Material S5. For each simulation setting, we select three different values of $\theta$, under which $\boldsymbol{X}_{4}$ is identified as a 


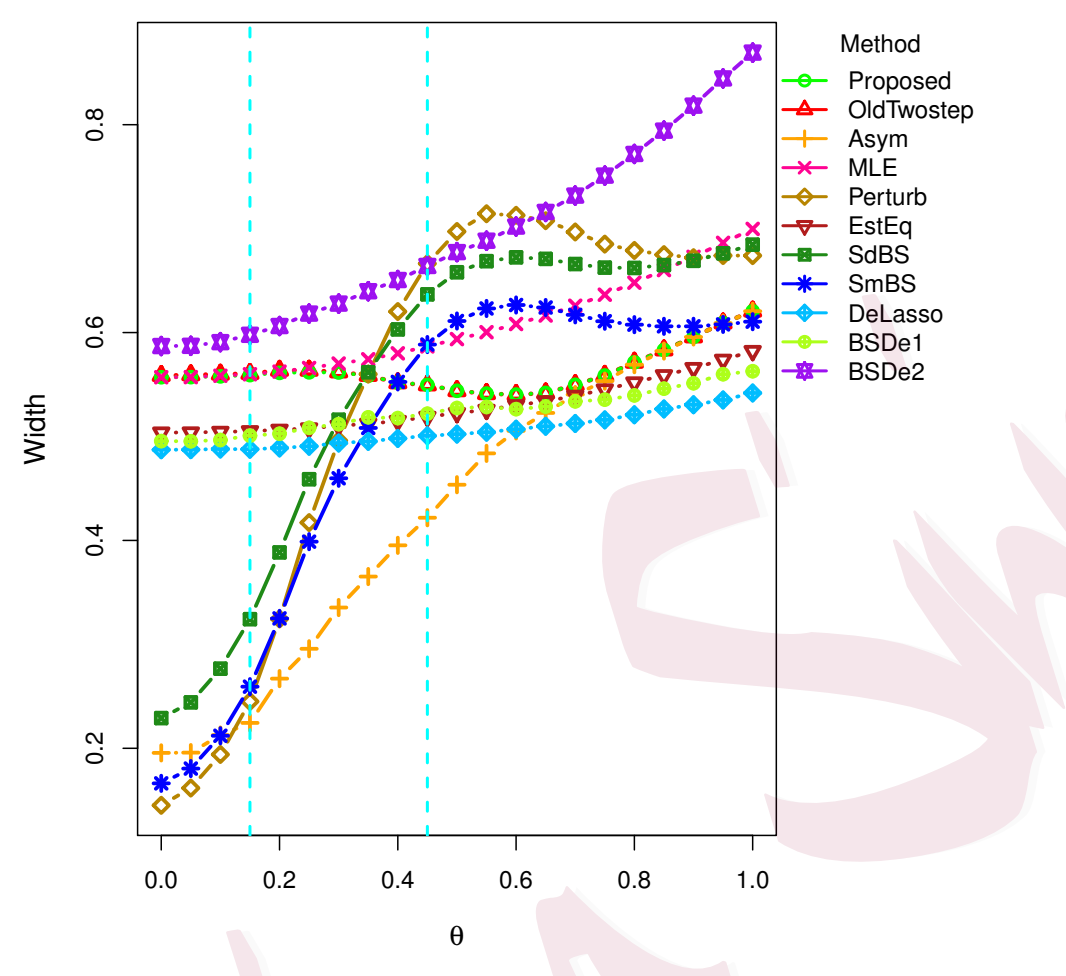

Figure 8: Average widths of the $95 \%$ confidence intervals when $(n, p, \rho)=(350,25,0)$. Proposed: the proposed two-step inference method; OldTwostep: the two-step inference method based on the idea of Shi and Qu (2017), which does not construct confidence intervals for identified noise variables; Asym: the method based on the asymptotic theory using the one-step adaptive Lasso estimator; MLE: the maximum likelihood estimation method; Perturb: the perturbation method; EstEq: the estimating equation-based method; SdBS: the standard bootstrap method; SmBS: the smoothed bootstrap method; DeLasso: the de-biased Lasso method; BSDe1: the type-I bootstrap de-biased Lasso method; BSDe2: the type-II bootstrap de-biased Lasso method.

noise variable, weak signal, and strong signal, respectively. In summary, the findings from the simulation setting of $(n, p, \rho)=(350,25,0)$ still hold under other simulation settings when $\rho=0$. By comparison, it can be found that the average widths of confidence intervals for all methods decrease with the sample size and increase with the correlations among covariates. When $\boldsymbol{X}_{4}$ is not a strong signal, regardless of the correlations among covariates, the confidence intervals for the asymptotic method have relatively low coverage probabilities. When $\boldsymbol{X}_{4}$ is a strong signal, if $\rho$ is 0 or 0.2 , the asymptotic method provides accurate confidence intervals, but if $\rho$ increases to 0.5 , the performance of the asymptotic method 
deteriorates. However, the coverage probabilities of confidence intervals for the proposed method are still close to $95 \%$ under all simulation settings.

In order to see whether the performance of the proposed method is sensitive to the choice of threshold values $\delta_{1}$ and $\tau$, we also consider other combinations of threshold values. For example, when $(n, p, \rho)=(350,25,0)$, we set $\tau$ as 0.1 and choose $\delta_{1}$ to be $0.96,0.97,0.98$ or 0.99, which is larger than $1-\alpha=0.95$. The empirical probabilities of assigning the covariate $\boldsymbol{X}_{4}$ to different signal categories are shown in Figure S5 of Supplementary Material S5. As the value of $\delta_{1}$ becomes larger and the value of $\theta$ is fixed, the empirical probability of identifying $\boldsymbol{X}_{4}$ as a weak signal becomes larger and the empirical probability of identifying $\boldsymbol{X}_{4}$ as a strong signal becomes smaller if $\theta$ is not sufficiently large, while the empirical probability of identifying $\boldsymbol{X}_{4}$ as a noise variable does not change. This is due to the proposed signal identification criterion. Figures S6-S7 in Supplementary Material S5 show the corresponding coverage probabilities and average widths of the $95 \%$ confidence intervals for the proposed two-step inference method, from which we can find that the coverage probability becomes larger as $\delta_{1}$ increases and $\theta$ is between 0.6 and 0.75 ; the average width becomes larger as $\delta_{1}$ increases and $\theta$ is between 0.15 and 0.75 . This is not surprising because when $\delta_{1}$ increases, the probability of using the maximum likelihood method to construct the confidence intervals becomes larger. As shown in Figures 7 and 8 , when $\theta$ is not too large, the coverage probability and average width of confidence interval based on the maximum likelihood method is higher than that based on the asymptotic method. However, as $\delta_{1}$ varies, the changes of coverage probability and average width are not large.

We also consider another situation where $\delta_{1}$ is set to be 0.99 and $\tau$ is chosen to be 0.05, 0.1, 0.15 or 0.2. Figure S8 in Supplementary Material S5 shows the empirical probabil- 
ities of assigning the covariate $\boldsymbol{X}_{4}$ to different signal categories in this situation, from which we can find that as $\tau$ increases, the empirical probability of identifying $\boldsymbol{X}_{4}$ as a weak signal is larger and the empirical probability of identifying $\boldsymbol{X}_{4}$ as a noise variable is smaller if $\theta$ is not too large, while the empirical probability of identifying $\boldsymbol{X}_{4}$ as a strong signal remains the same. This is consistent with the proposed signal selection criterion. However, since the proposed two-step inference method uses the same confidence interval construction method for the identified noise variables and weak signals, therefore the confidence interval does not change with the value of $\tau$, as shown in Figures S9-S10 of Supplementary Material S5.

We also examine whether the performance of the proposed method is sensitive to the total number of weak signals. We reset the regression coefficient vector $\boldsymbol{\beta}_{0}$ to be $(1,1,0.5, \theta, \underbrace{0.3, \ldots, 0.3}_{q}, \underbrace{0, \ldots, 0}_{p-q-4})^{\top}$, where $q$ is taken to be $0,1,2,3$. For illustration, let $(n, p, \rho)=(350,25,0), \delta_{1}$ be 0.99 and $\tau$ be 0.1 . Based on the signal identification criterion, all the $q$ covariates corresponding to the coefficient 0.3 are weak signals if $\theta$ ranges from 0 to 1 . If the covariate $\boldsymbol{X}_{4}$ is identified as a weak signal, then the total number of weak signals is $q+1$; otherwise $q$. The empirical probabilities of assigning the covariate $\boldsymbol{X}_{4}$ to different signal categories are shown in Figure S11 of Supplementary Material S5, which are not sensitive to the value of $q$. Figures S12-S13 in Supplementary Material S5 show the coverage probabilities and average widths of the $95 \%$ confidence intervals for the proposed two-step inference method, showing that when $\theta$ is small, the average width increases with the value of $q$, while the coverage probability does not change monotonously with the value of $q$. In addition, as $q$ varies, the variations of average width and coverage probability are not large. Thus, the performance of the proposed method is quite robust to the total number of weak signals. 


\section{Real data application}

To illustrate the performance of the proposed method, we apply it to a dataset in the Practice Fusion diabetes study, which was provided by Kaggle as part of the "Practice Fusion Diabetes Classification" challenge (Kaggle, 2012). The dataset consists of de-identified electronic medical records for over 10,000 patients. There are a total of 9948 patients in the training data, including a binary variable indicating whether a patient is diagnosed with Type 2 diabetes mellitus (T2DM) or not. In this analysis, we aim to determine the most important risk factors for the incidence of T2DM, which can be used to identify patients with a high risk of $\mathrm{T} 2 \mathrm{DM}$.

We first extract 119 predictors from the predictors selected by the first-place winner in the Kaggle competition by removing some highly correlated predictors (details can be found in https://www.kaggle.com/c/pf2012-diabetes/overview/winners). These predictors can be divided into six categories: basic information, transcript records, diagnosis information, medication information, lab result, and smoking status. The detailed information about these predictors can be found in Table S5 in Supplementary Material S6. One outlying patient is also removed in our analysis due to its inaccurate information on the predictors. All the predictors are standardized beforehand. We adopt the following logistic regression model to fit the dataset:

$$
P\left(y_{i}=1 \mid \mathbf{x}_{i}\right)=\frac{\exp \left(\alpha+\sum_{j=1}^{p} x_{i j} \beta_{j}\right)}{1+\exp \left(\alpha+\sum_{j=1}^{p} x_{i j} \beta_{j}\right)}, \quad i=1, \ldots, n
$$

where $p=119$ and $n=9947$.

We first obtain the one-step adaptive Lasso estimates of regression coefficients following 
the tuning parameter selection procedure given in Supplementary Material S4. We then identify whether a predictor is a strong signal, weak signal, or noise variable based on the criterion (3.4). Here, we choose $\delta_{1}$ to be 0.99 and $\tau$ to be 0.1 . From all the predictors, we identify 18 strong signals, 32 weak signals, and 69 noise variables. The 18 strong signals are also all selected by the one-step adaptive Lasso estimator, indicating consistency between our method and the one-step adaptive Lasso estimator for strong signal selection. Among the 32 weak signals, 24 of them are also selected by the one-step adaptive Lasso estimator, while the other 8 predictors are only identified by our method. These 8 additionally identified predictors include the numbers of times being diagnosed with herpes zoster, hypercholesterolemia, hypertensive heart disease, respiratory infection, sleep apnea, and joint pain, respectively; the number of transcripts for cardiovascular disease and the number of diagnoses per weighted year. The relationships between these 8 additional identified predictors and diabetes have also been studied by other researchers. For example, Papagianni et al. (2018) gave a review of the studies on associations between herpes zoster and diabetes mellitus, and found that herpes zoster and T2DM were likely to coexist for the same patient.

Later, we construct the $95 \%$ confidence intervals using our two-step inference method, together with all other comparison methods in Section 5. Figure 9 shows the average widths of confidence intervals for strong and weak signals. For both strong and weak signals, the widths of confidence intervals for the two types of two-step inference methods are the same. For strong signals, the proposed method and the asymptotic method provide the shortest confidence intervals. For weak signals, the widths of confidence intervals based on the proposed method are smaller than those based on the perturbation method, standard bootstrap method, smoothed bootstrap method, and two types of bootstrap de-biased Lasso methods. 


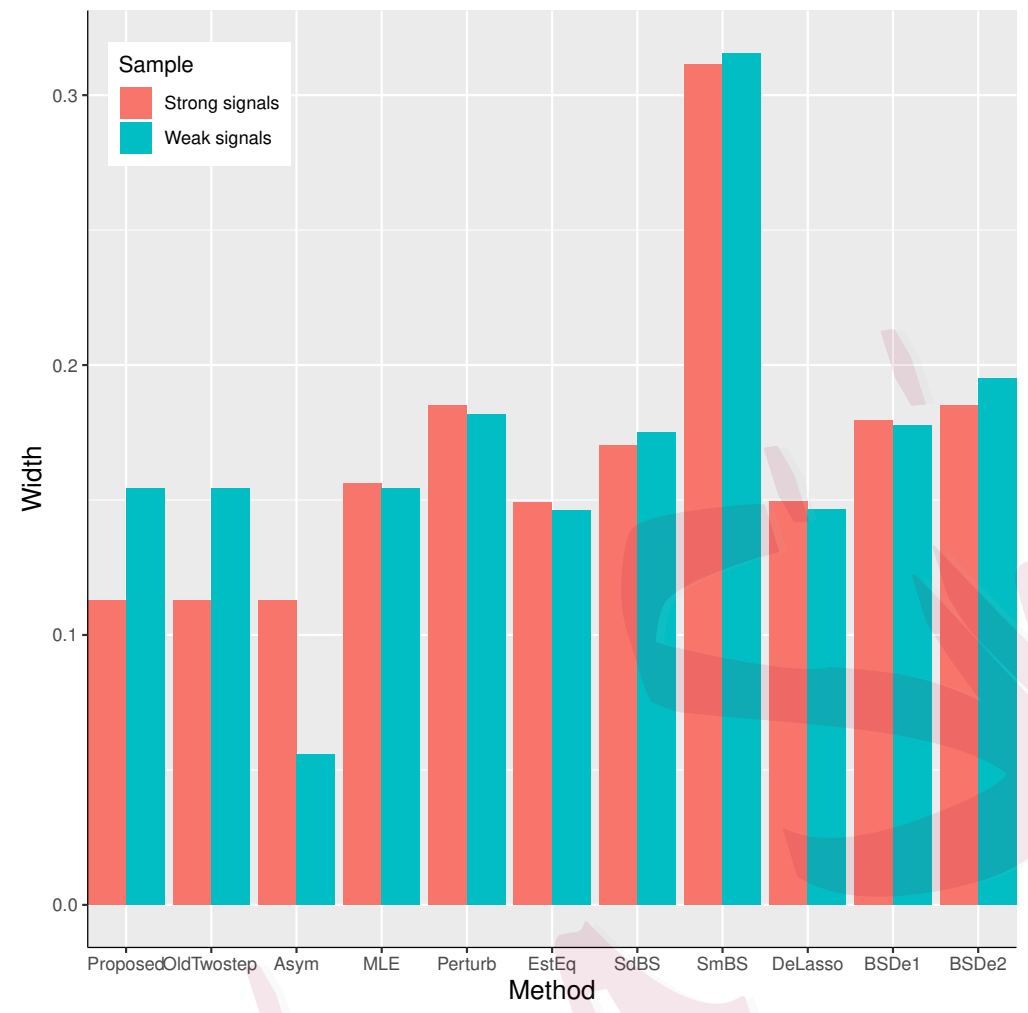

Figure 9: The average widths of the $95 \%$ confidence intervals for the diabetes dataset. Note that the asymptotic method does not construct confidence intervals for all the weak signals, the result for the weak signals is the average width of confidence intervals for the weak signals which are also selected by the asymptotic method. The meanings of notations: see Figures 6 and 7 .

\section{Discussion}

In this paper, we propose a new unified approach to weak signal identification and inference in penalized likelihood models, including the special case when the responses are categorical. To identify weak signals, we propose to use the estimated selection probability of each covariate as a measure of signal strength and develop a signal identification criterion based on the estimated selection probability directly. To construct confidence intervals for the regression coefficients, we propose a two-step inference procedure. Extensive simulation studies and a real data application show that the proposed signal identification method and two-step inference procedure outperform some existing ones in finite samples. 
The proposed method can also be extended to a high-dimensional setting where $p$ is not fixed. One possible way is to use the de-biased Lasso estimator as an initial estimator for the one-step adaptive Lasso estimator, and then leverage the asymptotic properties of the de-biased Lasso estimator to derive the selection probability. We can also use a penalized method to estimate the inverse of the information matrix, such as the CLIME estimator (Cai et al., 2011). In addition, our signal identification and inference framework can also be extended to longitudinal data. For longitudinal data, we can replace the negative loglikelihood function with the generalized estimating function in the estimation. Finally, in the fields of causal inference and econometrics, there is a popular "weak instrument" problem (Chao and Swanson, 2005; Burgess and Thompson, 2011; Choi et al., 2018), which can be considered as a weak signal problem. This is worth further development using our approach.

\section{Supplementary Materials}

The supplementary materials contain six sections. Section S1 provides details for the derivation of the approximated selection probability. Section S2 makes some additional detailed analysis of the approximated selection probability in finite samples. Section S3 shows the proof of Theorem 1. Section S4 presents implementation details of several methods. Sections S5 and S6 provide additional simulation results and information of the real data application, respectively.

\section{Acknowledgements}

This work was partially supported by the National Science Foundation of United States (DMS-1821198, DMS-1952406), National Natural Science Foundation of China (11671096, 11731011, 12071087), and Natural Sciences and Engineering Research Council of Canada 
(RGPIN-2019-07052, DGECR-2019-00453, RGPAS-2019-00093).

\section{References}

Burgess, S. and Thompson, S. G. (2011). Bias in causal estimates from mendelian randomization studies with weak instruments. Statistics in Medicine, 30(11):1312-1323.

Cai, T., Liu, W., and Luo, X. (2011). A constrained 1 minimization approach to sparse precision matrix estimation. Journal of the American Statistical Association, 106(494):594-607.

Chao, J. C. and Swanson, N. R. (2005). Consistent estimation with a large number of weak instruments. Econometrica, $73(5): 1673-1692$.

Choi, J., Gu, J., and Shen, S. (2018). Weak-instrument robust inference for two-sample instrumental variables regression. Journal of Applied Econometrics, 33(1):109-125.

Dezeure, R., Bühlmann, P., and Zhang, C.-H. (2017). High-dimensional simultaneous inference with the bootstrap. Test, 26(4):685-719.

Efron, B. (2014). Estimation and accuracy after model selection. Journal of the American Statistical Association, 109(507):991-1007.

Efron, B. and Tibshirani, R. J. (1994). An introduction to the bootstrap. CRC press.

Huang, J. and Xie, H. (2007). Asymptotic oracle properties of scad-penalized least squares estimators. In Asymptotics: Particles, processes and inverse problems, pages 149-166. Institute of Mathematical Statistics.

Javanmard, A. and Montanari, A. (2014). Confidence intervals and hypothesis testing for high-dimensional regression. The Journal of Machine Learning Research, 15(1):2869-2909. 
Jia, J., Xie, F., and Xu, L. (2019). Sparse poisson regression with penalized weighted score function. Electronic Journal of Statistics, 13(2):2898-2920.

Jin, J., Zhang, C.-H., and Zhang, Q. (2014). Optimality of graphlet screening in high dimensional variable selection. The Journal of Machine Learning Research, 15(1):2723-2772.

Kaggle (2012). Practice fusion diabetes classification. data retrieved from Kaggle competition dataset, http://www. kaggle.com/c/pf2012-diabetes

Lambert, P. and Eilers, P. H. (2005). Bayesian proportional hazards model with time-varying regression coefficients:

A penalized poisson regression approach. Statistics in Medicine, 24(24):3977-3989.

Li, Y., Hong, H. G., Ahmed, S. E., and Li, Y. (2019). Weak signals in high-dimensional regression: Detection, estimation and prediction. Applied Stochastic Models in Business and Industry, 35(2):283-298.

Liu, H., Xu, X., and Li, J. J. (2020). A bootstrap lasso+ partial ridge method to construct confidence intervals for parameters in high-dimensional sparse linear models. arXiv preprint arXiv:1706.02150.

Minnier, J., Tian, L., and Cai, T. (2011). A perturbation method for inference on regularized regression estimates. Journal of the American Statistical Association, 106(496):1371-1382.

Neykov, M., Ning, Y., Liu, J. S., Liu, H., et al. (2018). A unified theory of confidence regions and testing for high-dimensional estimating equations. Statistical Science, 33(3):427-443.

Papagianni, M., Metallidis, S., and Tziomalos, K. (2018). Herpes zoster and diabetes mellitus: a review. Diabetes Therapy, 9(2):545-550.

Park, M. Y. and Hastie, T. (2008). Penalized logistic regression for detecting gene interactions. Biostatistics, 9(1):3050. 
Reangsephet, O., Lisawadi, S., and Ahmed, S. E. (2020). Weak signals in high-dimensional logistic regression models. In International Conference on Management Science and Engineering Management, pages 121-133. Springer.

Shi, P. and Qu, A. (2017). Weak signal identification and inference in penalized model selection. The Annals of Statistics, 45(3):1214-1253.

Tibshirani, R. (1996). Regression shrinkage and selection via the lasso. Journal of the Royal Statistical Society: Series $B$ (Statistical Methodology), 58(1):267-288.

Tibshirani, R. (2011). Regression shrinkage and selection via the lasso: a retrospective. Journal of the Royal Statistical Society: Series B (Statistical Methodology), 73(3):273-282.

Tibshirani, R., Saunders, M., Rosset, S., Zhu, J., and Knight, K. (2005). Sparsity and smoothness via the fused lasso. Journal of the Royal Statistical Society: Series B (Statistical Methodology), 67(1):91-108.

Van de Geer, S., Bühlmann, P., Ritov, Y., Dezeure, R., et al. (2014). On asymptotically optimal confidence regions and tests for high-dimensional models. Annals of Statistics, 42(3):1166-1202.

Van de Geer, S., Bühlmann, P., and Zhou, S. (2011). The adaptive and the thresholded lasso for potentially misspecified models (and a lower bound for the lasso). Electronic Journal of Statistics, 5:688-749.

Wang, H. and Leng, C. (2007). Unified lasso estimation by least squares approximation. Journal of the American Statistical Association, 102(479):1039-1048.

Wu, T. T., Chen, Y. F., Hastie, T., Sobel, E., and Lange, K. (2009). Genome-wide association analysis by lasso penalized logistic regression. Bioinformatics, 25(6):714-721.

Yuan, M. and Lin, Y. (2006). Model selection and estimation in regression with grouped variables. Journal of the Royal Statistical Society: Series B (Statistical Methodology), 68(1):49-67. 
Zhang, C.-H. (2010). Nearly unbiased variable selection under minimax concave penalty. The Annals of Statistics, $38(2): 894-942$.

Zhang, C.-H. and Zhang, S. S. (2014). Confidence intervals for low dimensional parameters in high dimensional linear models. Journal of the Royal Statistical Society: Series B: Statistical Methodology, pages 217-242.

Zhang, H. and Jia, J. (2017). Elastic-net regularized high-dimensional negative binomial regression: consistency and weak signals detection. arXiv preprint arXiv:1712.03412.

Zhang, T. (2013). Multi-stage convex relaxation for feature selection. Bernoulli, 19(5B):2277-2293.

Zhang, Y. (2017). Recovery of weak signal in high dimensional linear regression by data perturbation. Electronic Journal of Statistics, 11(2):3226-3250.

Zhao, P. and Yu, B. (2006). On model selection consistency of lasso. Journal of Machine Learning Research, 7:25412563.

Zhu, J. and Hastie, T. (2004). Classification of gene microarrays by penalized logistic regression. Biostatistics, $5(3): 427-443$.

Zou, H. (2006). The adaptive lasso and its oracle properties. Journal of the American Statistical Association, 101(476):1418-1429.

Zou, H. and Hastie, T. (2005). Regularization and variable selection via the elastic net. Journal of the Royal Statistical Society: Series B (Statistical Methodology), 67(2):301-320.

Zou, H. and Li, R. (2008). One-step sparse estimates in nonconcave penalized likelihood models. Annals of Statistics, 36(4):1509-1533. 
Department of Computer and Mathematical Sciences, University of Toronto, ON M1C 1A4, Canada.

E-mail: yuexia.zhang@utoronto.ca

Facebook, Menlo Park, CA 94025, United States.

E-mail: pshi@fb.com

Department of Statistics, Fudan University, Shanghai 200433, China.

E-mail: zhuzy@fudan.edu.cn

Department of Statistical Sciences, University of Toronto, ON M5S 3G3, Canada.

E-mail: linbo.wang@utoronto.ca

Department of Statistics, University of California, Irvine, CA 92697, United States.

E-mail: aqu2@uci.edu 